\title{
On minimal forbidden subgraph characterizations of balanced graphs
}

\author{
Flavia Bonomo ${ }^{\mathrm{a}, \mathrm{b}}$, Guillermo Durán ${ }^{\mathrm{a}, \mathrm{c}, \mathrm{d}}$, Martín D. Safe ${ }^{\mathrm{e}, *}$, Annegret K. Wagler ${ }^{\mathrm{f}}$ \\ ${ }^{a}$ CONICET, Argentina \\ ${ }^{\mathrm{b}}$ Departamento de Computación, Facultad de Ciencias Exactas y Naturales, Universidad de Buenos Aires, Buenos Aires, Argentina \\ ${ }^{\mathrm{c}}$ Departamento de Matemática and Instituto de Cálculo, Facultad de Ciencias Exactas y Naturales, Universidad de Buenos Aires, \\ Buenos Aires, Argentina \\ d Departamento de Ingeniería Industrial, Facultad de Ciencias Físicas y Matemáticas, Universidad de Chile, Santiago, Chile \\ e Instituto de Ciencias, Universidad Nacional de General Sarmiento, Los Polvorines, Argentina \\ ${ }^{\mathrm{f}}$ CNRS and LIMOS, UFR Sciences et Technologies, Université Blaise Pascal, Clermont-Ferrand, France
}

\section{A R T I C L E I N F O}

\section{Article history:}

Received 23 March 2012

Received in revised form 9 December 2012

Accepted 1 April 2013

Available online 24 April 2013

\section{Keywords:}

Balanced graphs

Bipartite graphs

Hereditary clique-Helly graphs

Line graphs

Perfect graphs

\begin{abstract}
A B S T R A C T
A graph is balanced if its clique-matrix contains no edge-vertex incidence matrix of an odd chordless cycle as a submatrix. While a forbidden induced subgraph characterization of balanced graphs is known, there is no such characterization by minimal forbidden induced subgraphs. In this work, we provide minimal forbidden induced subgraph characterizations of balanced graphs restricted to graphs that belong to one of the following graph classes: complements of bipartite graphs, line graphs of multigraphs, and complements of line graphs of multigraphs. These characterizations lead to linear-time recognition algorithms for balanced graphs within the same three graph classes.
\end{abstract}

(c) 2013 Elsevier B.V. All rights reserved.

\section{Introduction}

A $\{0,1\}$-matrix is balanced [2] if it contains no edge-vertex incidence matrix of a chordless cycle with an odd number of vertices as a submatrix. Balanced matrices have remarkable properties studied in polyhedral combinatorics. Most notably, if $A$ is a balanced matrix, then $A$ is perfect and ideal, meaning, respectively, that the fractional set packing polytope $P(A)=$ $\left\{x \in \mathbb{R}^{n} \mid A x \leq \mathbf{1}, \mathbf{0} \leq x \leq \mathbf{1}\right\}$ and the fractional set covering polytope $Q(A)=\left\{x \in \mathbb{R}^{n} \mid A x \geq \mathbf{1}, \mathbf{0} \leq x \leq \mathbf{1}\right\}$ are integral (i.e., all their extreme points have integer coordinates) [10].

A graph is perfect if and only if its clique-matrix is perfect [8]. Here, a clique is an inclusion-wise maximal set of pairwise adjacent vertices and given an enumeration $Q_{1}, \ldots, Q_{k}$ of all cliques and an enumeration $v_{1}, \ldots, v_{n}$ of all vertices of a graph $G$, a clique-matrix of $G$ is the $k \times n\{0,1\}$-matrix $A=\left(a_{i j}\right)$ such that $a_{i j}=1$ if and only if $v_{j} \in Q_{i}$. Some years ago, the minimal forbidden induced subgraphs for the class of perfect graphs were identified [7], settling affirmatively a conjecture posed more than 40 years before by Berge [1]. They are the chordless cycles of odd length at least 5 , called odd holes, and their complements, the odd antiholes.

Theorem 1 (Strong Perfect Graph Theorem [7]). A graph is perfect if and only if it has no odd holes and no odd antiholes.

\footnotetext{
* Corresponding author. Tel./fax: +54 1144697506.

E-mail addresses: fbonomo@dc.uba.ar (F. Bonomo), gduran@dm.uba.ar (G. Durán), msafe@ungs.edu.ar (M.D. Safe), wagler@isima.fr (A.K. Wagler).
} 


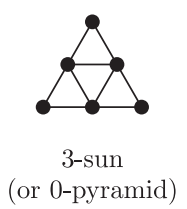

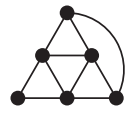

1-pyramid

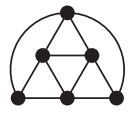

2-pyramid

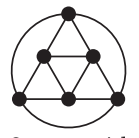

3-pyramid

Fig. 1. The pyramids.
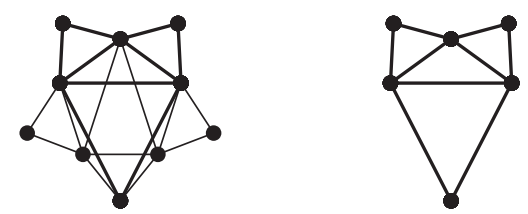

Fig. 2. An extended odd sun that is not minimal is shown on the left. Bold lines correspond to the edges of a proper induced extended odd sun, depicted on the right.

In analogy to perfect graphs, balanced graphs can be defined as those graphs whose clique-matrix is balanced. Since balanced matrices are perfect, balanced graphs form a subclass of the class of perfect graphs. The name 'balanced graphs' appears explicitly in [4], but these graphs were already considered in [2] (see Theorem 5 therein). Moreover, from Proposition 7 in [2], it follows that balanced graphs also belong to another interesting class, the class of hereditary clique-Helly graphs; i.e., the class of graphs whose induced subgraphs satisfy that the intersection of any nonempty family of pairwise intersecting cliques is nonempty [24]. Prisner characterized hereditary clique-Helly graphs both by their clique-matrices and by minimal forbidden induced subgraphs as follows.

Theorem 2 ([24]). A graph is hereditary clique-Helly if and only if its clique-matrix contains no edge-vertex incidence matrix of a cycle on three vertices as a submatrix or, equivalently, if and only if it does not contain any of the graphs in Fig. 1 as an induced subgraph.

Balanced graphs were characterized by means of forbidden induced subgraphs as follows. For a graph $G$ and a nonempty set $W \subseteq V(G)$, let $N(W)=\bigcap_{w \in W} N(w)$ be the set of vertices of $G$ being adjacent to all vertices of $W$, whereas $N(\emptyset)=V(G)$. For each edge $e=u v$, let $N(e)$ be a shorthand for $N(\{u, v\})$. An unbalanced cycle of $G$ is an odd cycle $C$ such that, for each edge $e \in E(C)$ (i.e., joining two consecutive vertices of $C$ ), there exists a (possibly empty) complete subgraph $W_{e}$ of $G$ such that $W_{e} \subseteq N(e) \backslash V(C)$ and $N\left(W_{e}\right) \cap N(e) \cap V(C)=\emptyset$. Notice that it is possible for the sets $W_{e}$ and $W_{e^{\prime}}$ for different edges $e$ and $e^{\prime}$ to have nonempty intersection. An extended odd sun is a graph $G$ with an unbalanced cycle $C$ such that $V(G)=V(C) \cup \bigcup_{e \in E(C)} W_{e}$ and $\left|W_{e}\right| \leq|N(e) \cap V(C)|$ for each edge $e \in E(C)$. The extended odd suns with the smallest number of vertices are $C_{5}$ and the pyramids in Fig. 1. Moreover, every odd hole is an extended odd sun (by letting $W_{e}=\emptyset$ for each $e$ ). Notice that $C_{3}$ is not an extended odd sun, since otherwise we would be forced to choose $W_{e}=\emptyset$ for each edge $e$, but then $N\left(W_{e}\right) \cap N(e) \cap V(C)=\{v\}$ where $v$ is the only vertex non-incident to $e$ (because $N\left(W_{e}\right)=N(\emptyset)=V(C)$ and $N(e)=\{v\})$. The characterization of balancedness by forbidden induced subgraphs is as follows.

Theorem 3 ([2,5]). A graph is balanced if and only if it has no unbalanced cycle or, equivalently, if and only if it contains no induced extended odd sun.

However, the above characterization is not by minimal forbidden induced subgraphs because some extended odd suns contain some other extended odd suns as proper induced subgraphs, as Fig. 2 shows. In this work, we address the problem of characterizing balanced graphs by minimal forbidden induced subgraphs.

Bipartite graphs, complements of bipartite graphs, line graphs of bipartite graphs, and complements of line graphs of bipartite graphs are well-known classes of perfect graphs. Their perfectness follows already from the works of Kőnig $[17,18]$. Moreover, these four graph classes constitute four of the five basic perfect graph classes in the decomposition of perfect graphs devised for the proof of the Strong Perfect Graph Theorem [7].

Bipartite graphs and line graphs of bipartite graphs are also balanced [3], but not necessarily their complements. This is due to the fact that, contrary to perfect graphs, balanced graphs are not closed under complementation. For example, the graphs in Fig. 1 are not balanced but have balanced complements. In this paper we present minimal forbidden induced subgraph characterizations of balanced graphs restricted to complements of bipartite graphs, line graphs of multigraphs, and complements of line graphs of multigraphs. It turns out that, for the first two studied classes, a member of these classes is balanced if and only if it is perfect and hereditary clique-Helly.

In addition, we address the problem of recognizing balanced graphs efficiently within the same three graph classes. Let $G$ be a given graph with $n$ vertices and $m$ edges. In [6], it is shown that it can be decided whether or not $G$ is perfect in $O\left(n^{9}\right)$ time. It is easy to see that it can be decided whether or not $G$ is balanced in $O\left(m^{9}+n\right)$ time by first computing a clique-matrix of $G$ and then testing this matrix for balancedness, as follows. As a graph is balanced if and only if each of its connected components is balanced, we assume, without loss of generality, that $G$ is connected. To compute the clique-matrix 


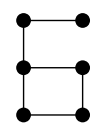

E

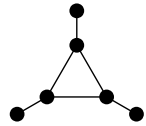

net

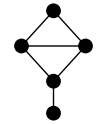

kite

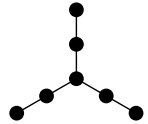

bipartite claw

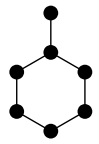

6-pan

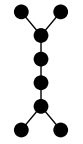

braid

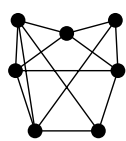

$U_{7}$

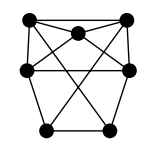

$V_{7}$

Fig. 3. Some small graphs.

of $G$ we can rely on the algorithm in [28] that enumerates the cliques of $G$, one after the other, in $O(m n)$ time per clique. If $G$ is balanced, then it is also hereditary clique-Helly and, as a result, it has at most $m$ cliques [24,29]. Consequently, we can, in $O\left(m^{2} n\right)$ time, either compute a clique-matrix of $G$ or detect that $G$ is not hereditary clique-Helly and, in particular, not balanced. Finally, we can decide whether $G$ is balanced by testing its clique-matrix for balancedness in $O\left(\mathrm{~m}^{9}\right)$ time by means of the recognition algorithm for balanced matrices in [31]. As a consequence of our structural characterizations, we will show that the problem of recognizing balanced graphs is linear-time solvable within complements of bipartite graphs, line graphs of multigraphs, and complements of line graphs of multigraphs.

The paper is organized as follows. The next subsection provides all basic definitions from graph theory used in what follows. In Sections 2-4, we characterize balanced graphs within complements of bipartite graphs, line graphs of multigraphs, and complements of line graphs of multigraphs, respectively, including characterizations by minimal forbidden induced subgraphs. In each of these sections, we first provide the definition and crucial properties of the graph class within which we study balanced graphs and then present our structural characterizations and discuss the recognition of balancedness within this class.

\subsection{Basic definitions}

Graphs in this paper are finite, undirected, without loops, and without multiple edges. We will also deal with multigraphs, introduced near the end of this subsection. The cardinality of any set $S$ will be denoted by $|S|$.

Let $G$ be a graph. We denote the vertex set of $G$ by $V(G)$, its edge set by $E(G)$, and its complement by $\bar{G}$. The neighborhood of a vertex $v$ in $G$ is the set $N_{G}(v)$ consisting of all the vertices of $G$ that are adjacent to $v$. The common neighborhood of an edge $e=v w$ is $N_{G}(e)=N_{G}(v) \cap N_{G}(w)$ and, in general, the common neighborhood of a nonempty set $W \subseteq V(G)$ is $N_{G}(W)=\bigcap_{w \in W} N_{G}(w)$, whereas $N_{G}(\emptyset)=V(G)$. An isolated vertex is a vertex with no neighbors, a pendant vertex is a vertex with precisely one neighbor, and a universal vertex is a vertex adjacent to every other vertex of the graph. The degree of $v$ in $G$ is $d_{G}(v)=\left|N_{G}(v)\right|$. Two vertices $v, w$ are false twins in $G$ if they are nonadjacent and $N_{G}(v)=N_{G}(w)$, while they are true twins in $G$ if they are false twins in $\bar{G}$.

Let $G$ be a graph. A subgraph of $G$ is a graph $H$ such that $V(H) \subseteq V(G)$ and $E(H) \subseteq E(G)$. Let $H_{1}$ and $H_{2}$ be two subgraphs of $G$. We say that $H_{1}$ and $H_{2}$ touch if they share exactly one vertex of $G$. Moreover, if $V\left(H_{1}\right) \cap V\left(H_{2}\right)=\{v\}$, we say that $H_{1}$ and $H_{2}$ touch at $v$. If $W \subseteq V(G)$, the subgraph induced by $W$ in $G$ is denoted by $G[W]$ and $G-W$ denotes $G[V(G) \backslash W]$. If $W=\{w\}$, we denote $G-W$ simply by $G-w$. Let $H$ be a graph. We say that $G$ contains $H$ if $H$ is isomorphic to a subgraph of $G$ (either induced or not), and we say that $G$ contains an induced $H$ if $H$ is isomorphic to an induced subgraph of $G$. A class $g$ of graphs is hereditary if, for each graph $G$ of $g$, every induced subgraph of $G$ also belongs to $g$. The class of balanced graphs is hereditary because a clique-matrix of an induced subgraph of a graph $G$ is a submatrix of some clique-matrix of $G$ (cf. [5]). We say that $G$ is $H$-free to mean that $G$ contains no induced $H$. If $\mathscr{H}$ is a collection of graphs, we say that $G$ is $\mathscr{H}$-free to mean that $G$ contains no induced $H$ for any $H \in \mathscr{H}$. Some special small graphs to be referred in what follows are depicted in Fig. 3. We will call any of the graphs in Fig. 1 a pyramid.

A complete is a set of pairwise adjacent vertices. The complete graph on $n$ vertices will be denoted by $K_{n}$. A complete on three vertices is called a triangle. An inclusion-wise maximal complete is a clique. A stable set of a graph is a set of pairwise nonadjacent vertices.

Paths and cycles are assumed to be simple; i.e., with no repeated vertices aside from the starting and ending vertices in the case of cycles. An n-path (resp. $n$-cycle) is a path (resp. cycle) on $n$ vertices. The cycles on three vertices are also called triangles. The starting and ending vertices of a path are called the endpoints of the path. Let $Z$ be a path or a cycle. By the edges of $Z$ we mean those edges joining two consecutive vertices of $Z$. The set of edges of $Z$ will be denoted by $E(Z)$ and the length of $Z$ is $|E(Z)|$. The distance between two vertices is the minimum length of a path joining them. A chord of $Z$ is an edge whose endpoints are nonconsecutive vertices of $Z$. The chordless $n$-cycle is denoted by $C_{n}$ and the chordless $n$-path is denoted by $P_{n}$. A chord of a cycle is short if its endpoints are at distance two within the cycle, and is long otherwise. A cycle is odd (resp. even) if it has an odd (resp. even) number of vertices. A hole is a chordless cycle of length at least 4 . An antihole is the complement of a hole of length at least 5.

A graph $G$ is connected if any pair of its vertices is linked by a path. A connected graph without cycles is a tree. A component is an inclusion-wise maximal connected subgraph. For an edge $e$ of $G$, we denote by $G-e$ the graph that arises from $G$ by making the endpoints of $e$ nonadjacent. We say that an edge $e$ of a graph $G$ is a bridge if $G-e$ has more components than $G$. If $v$ and $w$ are two nonadjacent vertices of $G$, then $G+v w$ denotes the graph that arises from $G$ by making $v$ and $w$ adjacent. 


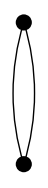

$\mathrm{C}_{2}$

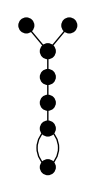

1-braid

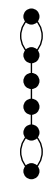

2-braid

Fig. 4. Some special multigraphs.

Multigraphs are an extension of graphs obtained by allowing different edges to join the same pair of vertices. Multigraphs are still finite, undirected, and without loops. Two edges joining the same pair of vertices are called parallel. We denote the vertex set of a multigraph $H$ by $V(H)$ and its edge set by $E(H)$. If $H$ is a multigraph, the underlying graph of $H$ is the graph $\widehat{H}$ having the same vertices as $H$ and two vertices of $\widehat{H}$ are adjacent if there is at least one edge in $H$ joining them. If $v$ is a vertex of a multigraph $H$, we denote by $\hat{d}_{H}(v)$ the degree of $v$ in the underlying graph $\widehat{H}$. A vertex of a multigraph is pendant if it has exactly one neighbor; i.e., if it is a pendant vertex of the underlying graph. Notice that there may be many edges joining a pendant vertex to its only neighbor.

We say that $H^{\prime}$ is a submultigraph of $H$ if $V(H) \subseteq V\left(H^{\prime}\right)$ and, for each pair of adjacent vertices $u$ and $v$ of $H^{\prime}$, there are at least as many edges in $H$ joining them as there are in $H^{\prime}$. We say that $H^{\prime}$ is contained in $H$ or that $H$ contains $H^{\prime}$ if and only if $H^{\prime}$ is isomorphic to a submultigraph of $H^{\prime}$. Two submultigraphs touch at vertex $v$ if $v$ is their only common vertex. A multigraph is connected if its underlying graph is connected and a component of a multigraph is an inclusion-wise maximal connected submultigraph.

The paths and cycles of a multigraph are the paths and cycles of its underlying graph. A multitree is a connected multigraph without cycles; i.e., a multigraph whose underlying graph is a tree. Some multigraphs needed in what follows are displayed in Fig. 4. Notice that we denote the multigraph consisting of two vertices and two parallel edges joining them by $C_{2}$, despite not being a cycle under our definition.

Let $H_{1}$ and $H_{2}$ be two vertex-disjoint graphs or multigraphs. The disjoint union of $H_{1}$ and $H_{2}$ has vertex set $V\left(H_{1}\right) \cup V\left(H_{2}\right)$ and two vertices $u$ and $v$ are adjacent in $H$ if and only if they are adjacent in $H_{i}$ for some $i \in\{1,2\}$ and there are exactly as many edges joining $u$ and $v$ in $H$ as there are in $H_{i}$. If $t$ is a nonnegative integer and $H$ is a multigraph, $t H$ denotes the disjoint union of $t$ copies of $H$.

\section{Balancedness of complements of bipartite graphs}

A graph is bipartite if its vertex set can be partitioned into two stable sets $X$ and $Y$. If so, $\{X, Y\}$ is called a bipartition. If, in addition, every vertex of $X$ is adjacent to every vertex of $Y$, the graph is complete bipartite.

Recall that bipartite graphs are balanced, but that the class of balanced graphs is not self-complementary, and the complements of bipartite graphs are not necessarily balanced. In this subsection, we characterize those complements of bipartite graphs that are balanced by minimal forbidden induced subgraphs. In fact, we show that the complement of a bipartite graph is balanced if and only if it is hereditary clique-Helly.

Theorem 4. Let $G$ be the complement of a bipartite graph. Then, the following statements are equivalent:

(i) $G$ is balanced.

(ii) A clique-matrix of $G$ contains no edge-vertex incidence matrix of $C_{3}$.

(iii) $G$ is hereditary clique-Helly.

(iv) G contains no induced 1-pyramid, 2-pyramid, or 3-pyramid.

Proof. That (i) $\Rightarrow$ (ii) $\Rightarrow$ (iii) $\Rightarrow$ (iv) follows from the discussion in the introduction. In order to prove that (iv) $\Rightarrow$ (i), assume that $G$ contains no induced 1-pyramid, 2-pyramid, or 3-pyramid, and we will prove that $G$ is balanced. Since $G$ is the complement of a bipartite graph, its vertex set can be partitioned into red and blue vertices such that any two vertices of the same color are adjacent. Suppose, for the purpose of contradiction, that $G$ is not balanced. Let $C=v_{1} v_{2} \ldots v_{2 t+1} v_{1}$ be an unbalanced cycle in $G$ and let the $W_{e}$ 's for each $e \in E(C)$ be accordingly. Since the 3-sun is not the complement of a bipartite graph, $G$ is pyramid-free and, therefore, $t>1$.

Since $C$ is odd, there exist consecutive vertices $v_{k}$ and $v_{k+1}$ in $C$ having the same color. Either there is another vertex $v_{\ell}$ in $C \backslash\left\{v_{k}, v_{k+1}\right\}$ of this color, or all vertices in $C \backslash\left\{v_{k}, v_{k+1}\right\}$ have the other color. In any case, $C$ has three pairwise different vertices $v_{i}, v_{i+1}$, and $v_{j}$ of the same color, say red. Thus, $v_{i}, v_{i+1}$, and $v_{j}$ induce a triangle and $v_{j} \in N\left(v_{i} v_{i+1}\right) \cap V(C)$ follows.

Next, we shall construct a blue triangle $u_{1}, u_{2}$, and $u_{3}$ in G. By the definition of an unbalanced cycle, $N\left(W_{v_{i} v_{i+1}}\right) \cap$ $N\left(v_{i} v_{i+1}\right) \cap V(C)=\emptyset$ and there exists some $u_{1} \in W_{v_{i} v_{i+1}}$ such that $u_{1}$ is nonadjacent to $v_{j}$. Since $v_{j}$ is red, $u_{1}$ is blue. If $v_{i-1}$ is nonadjacent to $v_{i+1}$, we let $u_{2}=v_{i-1}$; otherwise, $v_{i+1} \in N\left(v_{i-1} v_{i}\right) \cap V(C)$ and we let $u_{2}$ be any vertex of $W_{v_{i-1} v_{i}}$ nonadjacent to $v_{i+1}$. In both cases, $u_{2}$ is blue because it is nonadjacent to the red vertex $v_{i+1}$. Similarly, if $v_{i+2}$ is nonadjacent to $v_{i}$, we define $u_{3}=v_{i+2}$. Otherwise, we let $u_{3}$ be any vertex of $W_{v_{i+1} v_{i+2}}$ nonadjacent to $v_{i}$. In both cases, $u_{3}$ is blue because it is nonadjacent to $v_{i}$. By construction, $u_{1}, u_{2}$, and $u_{3}$ are pairwise different because $N_{G}\left(u_{1}\right) \cap\left\{v_{i}, v_{i+1}\right\}=\left\{v_{i}, v_{i+1}\right\}, N_{G}\left(u_{2}\right) \cap\left\{v_{i}, v_{i+1}\right\}=\left\{v_{i}\right\}$, and $N_{G}\left(u_{3}\right) \cap\left\{v_{i}, v_{i+1}\right\}=\left\{v_{i+1}\right\}$. Since $u_{1}, u_{2}$, and $u_{3}$ are blue, they induce a triangle in $G$. Therefore, $\left\{u_{1}, v_{i}, v_{i+1}, v_{j}, u_{2}, u_{3}\right\}$ 
induces a 1-pyramid, 2-pyramid, or 3-pyramid in $G$, a contradiction. Hence, $G$ is balanced, which concludes the proof of (iv) $\Rightarrow$ (i).

As a consequence of the equivalence between (i) and (iii) of the above theorem, deciding if the complement of a bipartite graph is balanced is equivalent to deciding whether it is hereditary clique-Helly. The currently best time bound for recognizing hereditary clique-Helly graphs is $O\left(\mathrm{~m}^{2}\right)$ where $m$ is the number of edges of the input graph [20]. Notice that if the input graph is the complement of a bipartite graph with $n$ vertices and $m$ edges, then $m^{2}=\Theta\left(n^{4}\right)$, which means that $O\left(\mathrm{~m}^{2}\right)$ is not a linear-time bound. We will show that there is a simple linear-time recognition algorithm for hereditary clique-Helly graphs (or, equivalently, balanced graphs) when the input graph is known to be the complement of a bipartite graph.

A component is nontrivial if it has at least two vertices, and is trivial otherwise. Consider the following consequence of Theorem 4.

Corollary 5. Let $G$ be the complement of a bipartite graph. Then, $G$ is balanced if and only if one of the following conditions holds:

(i) $\overline{\bar{G}}$ has only trivial components.

(ii) $\bar{G}$ has exactly one nontrivial component, which is $\left\{E, P_{4} \cup P_{2}, 3 K_{2}\right\}$-free.

(iii) $\bar{G}$ has exactly two nontrivial components, each of which is a complete bipartite graph.

Proof. Let $G$ be the complement of a bipartite graph. If $\bar{G}$ has only trivial components, then $G$ is a complete graph and, in particular, $G$ is balanced. If $\bar{G}$ has more than two nontrivial components, then $G$ contains an induced 3-pyramid and, consequently, $G$ is not balanced. Notice that, because of Theorem $4, G$ is balanced if and only if $\bar{G}$ is $\left\{E, P_{4} \cup P_{2}, 3 K_{2}\right\}$-free. Thus, if we assume that $\bar{G}$ has exactly one nontrivial component, then $G$ is balanced if and only if (ii) holds. Consequently, it only remains to prove the validity of the corollary in the case where $\bar{G}$ has exactly two nontrivial components.

Suppose that $G$ has exactly two nontrivial components $\bar{G}_{1}$ and $\bar{G}_{2}$. We must prove that $G$ is balanced if and only if $\bar{G}_{1}$ and $\bar{G}_{2}$ are complete bipartite graphs. By Theorem 4, it is enough to prove that $\bar{G}$ is $\left\{E, P_{4} \cup P_{2}, 3 K_{2}\right\}$-free if and only if $\bar{G}_{1}$ and $\bar{G}_{2}$ are complete bipartite graphs. Clearly, if $\bar{G}_{1}$ and $\bar{G}_{2}$ are complete bipartite graphs, then $\bar{G}$ is $\left\{E, P_{4} \cup P_{2}, 3 K_{2}\right\}$-free because $\bar{G}$ is the disjoint union of $\bar{G}_{1}$ and $\bar{G}_{2}$ and each of them is $\left\{E, P_{4}, 2 K_{2}\right\}$-free. Conversely, assume that $\bar{G}$ is $\left\{E, P_{4} \cup P_{2}, 3 K_{2}\right\}$-free. Since $\bar{G}_{1}$ and $\bar{G}_{2}$ are nontrivial and $\bar{G}$ is $\left(P_{4} \cup P_{2}\right)$-free, $\bar{G}_{1}$ and $\bar{G}_{2}$ are $P_{4}$-free. Thus, for each $i \in\{1,2\}$, each two nonadjacent vertices of $\bar{G}_{i}$ must be on the same set of the bipartition of $\bar{G}_{i}$; i.e., $\bar{G}_{1}$ and $\bar{G}_{2}$ are complete bipartite graphs.

Let $G$ be the complement of a bipartite graph $H, n$ and $m$ the number of vertices and edges of $G$. We will show that there is a simple $O\left(n^{2}\right)$-time algorithm that decides whether $G$ is balanced or not. Notice that $O\left(n^{2}\right)$ is here a linear-time bound because, $G$ being the complement of a bipartite graph, $m=\Theta\left(n^{2}\right)$. Since conditions (i) and (iii) of Corollary 5 can be clearly verified in $O\left(n^{2}\right)$ time, it only remains to show that it can be decided in $O\left(n^{2}\right)$ time whether or not a connected bipartite graph is $\left\{E, P_{4} \cup P_{2}, 3 K_{2}\right\}$-free.

For a connected bipartite graph $H=(X, Y ; F)$ with bipartition $\{X, Y\}$ and edge set $F$, its bipartite complement is the bipartite graph $\bar{H}^{\text {bip }}=(X, Y ;(X \times Y) \backslash F)$. For instance, $\bar{P}_{5}^{\text {bip }}=2 K_{2} \cup K_{1}$. We will show that a recognition algorithm for $\left\{E, P_{4} \cup P_{2}, 3 K_{2}\right\}$-free bipartite graphs follows from the study of $E$-free bipartite graphs in [22] using the following result.

Theorem 6 ([22]). Let $H$ be a connected bipartite graph. Then, the following assertions are equivalent:

(i) $\mathrm{H}$ is $\left\{E, P_{7}\right\}$-free.

(ii) $\mathrm{H}$ is $\overline{P_{5}}$-free.

(iii) Each component of $\bar{H}^{\text {bip }}$ is $2 K_{2}$-free.

We have the following immediate consequence.

Corollary 7. Let $H$ be a connected bipartite graph. Then $H$ is $\left\{E, P_{4} \cup P_{2}, 3 K_{2}\right\}$-free if and only if each component of $\bar{H}^{\text {bip }}$ is $2 K_{2}$-free.

Proof. In fact, if $H$ is $\left\{E, P_{4} \cup P_{2}, 3 K_{2}\right\}$-free, then, in particular, $H$ is $\left\{E, P_{7}\right\}$-free (because $P_{7}$ contains an induced $P_{4} \cup P_{2}$ ) and, by Theorem 6 , each component of $\bar{H}^{\text {bip }}$ is $2 K_{2}$-free.

Conversely, suppose that each component of $\bar{H}^{\text {bip }}$ is $2 K_{2}$-free. Then, by Theorem $6, H$ is ${\overline{P_{5}}}^{\text {bip }}$-free. Since each of $E, P_{4} \cup P_{2}$, and $3 K_{2}$ contains an induced $\overline{P_{5}}$ bip,$H$ is $\left\{E, P_{4} \cup P_{2}, 3 K_{2}\right\}$-free.

Bipartite $2 K_{2}$-free graphs are known as chain graphs [30] or difference graphs [13] and it is well known that they can be recognized in linear time (see, e.g., [14]). Therefore, as a consequence of Corollary 7, given a connected bipartite graph $H$ with $n$ vertices, it can be decided whether $H$ is $\left\{E, P_{4} \cup K_{2}, 3 K_{2}\right\}$-free in $O\left(n^{2}\right)$ time. Indeed, $\bar{H}^{\text {bip }}$ can be clearly computed in $O\left(n^{2}\right)$ time and, since bipartite chain graphs can be recognized in linear time, we can decide whether each of the components of $\bar{H}^{\text {bip }}$ is $2 K_{2}$-free also in $O\left(n^{2}\right)$ time.

Altogether, we have an $O\left(n^{2}\right)$-time algorithm to decide the balancedness or not of any given complement of a bipartite graph with $n$ vertices. Recalling that an $O\left(n^{2}\right)$-time algorithm is linear-time if its input is the complement of a bipartite graph, we conclude the following.

Corollary 8. It can be decided in linear time whether or not the complement of a bipartite graph is balanced (or, equivalently, hereditary clique-Helly). 


\section{Balancedness of line graphs of multigraphs}

For a graph or multigraph $R$, its line graph $L(R)$ is obtained by taking one vertex for each edge of $R$ and joining two vertices in $L(R)$ if the corresponding edges of $R$ share at least one endpoint. Conversely, a graph $G$ is a line graph of a multigraph if there is some multigraph $R$ such that $G=L(R)$; if $R$ can be chosen to be a graph, $G$ is simply said to be a line graph.

It is known that line graphs of bipartite graphs are balanced and which line graphs are perfect [27]. We prove structural characterizations of those line graphs that are balanced, including a characterization by minimal forbidden induced subgraphs, and show how these structural results naturally extend to line graphs of multigraphs.

In order to state our results we need to introduce some definitions. First, we note that the cliques in a line graph $L(R)$ correspond to maximal sets of pairwise incident edges in $R$, called the $L$-cliques of $R$ : that are the edge sets of triangles, called triads, and the stars $S_{R}(v)$ consisting of all edges incident to a vertex $v$ which are not contained in another star or a triad.

A $t$-bloom $\left\{v ; v_{1}, \ldots, v_{t}\right\}$ in a graph is a set of $t>0$ different pendant vertices $v_{1}, \ldots, v_{t}$ all being adjacent to vertex $v$. By contracting two nonadjacent vertices $u$ and $v$ we replace them by a new vertex $w$ with $N(w)=N(u) \cup N(v)$. Let $G_{1}$ and $G_{2}$ be two vertex-disjoint graphs and $A=\left\{a ; a_{1}, \ldots, a_{t}\right\}$ a $t$-bloom in $G_{1}, B=\left\{b ; b_{1}, \ldots, b_{t}\right\}$ a $t$-bloom in $G_{2}$, then $G_{1} \triangle_{A B} G_{2}$ denotes the graph that arises from $G_{1} \cup G_{2}$ by adding the edge $a b$ and contracting $a_{i}$ with $b_{i}$ for each $i=1, \ldots, t$.

The following result characterizes which line graphs are balanced, including a characterization by minimal forbidden induced subgraphs.

Theorem 9. For a graph $R$ and its line graph $G=L(R)$, the following assertions are equivalent:

(i) $G$ is balanced.

(ii) $G$ is perfect and hereditary clique-Helly.

(iii) G has no odd holes and contains no induced 3-sun, 1-pyramid, or 3-pyramid.

(iv) $R$ has no odd cycles of length at least 5 and contains no net, kite, or $K_{4}$.

(v) Each component of $R$ belongs to the graph class $\&$ which is the minimal graph class satisfying the following two conditions:

(a) All connected bipartite graphs belong to 8 .

(b) If $G_{1}, G_{2} \in s$ and the sets $A$ and $B$ are t-blooms of $G_{1}$ and $G_{2}$, respectively, then $G_{1} \triangle_{A B} G_{2}$ belongs to \&.

Proof. The implications (i) $\Rightarrow$ (ii) $\Rightarrow$ (iii) follow from the discussion in the introduction. That (iii) $\Rightarrow$ (iv) follows from the definition of line graphs.

We prove that (iv) $\Rightarrow(v)$ by induction on the number $n$ of edges of $R$. Assume that $R$ has no odd cycles of length at least 5 and contains no kite, net, or $K_{4}$. If $n=1$, (v) holds trivially. Let $n>1$ and assume that (v) holds for graphs with less than $n$ edges. Let $S$ be any component of $R$ and assume that $S$ is not bipartite. In order to prove that $S$ belongs to $\delta$, we need to show that $S=S_{1} \triangle_{A B} S_{2}$ for some $S_{1}, S_{2} \in \delta$ and some blooms $A$ and $B$. Since $S$ is not bipartite and has no odd cycles of length at least 5 , there is some triangle $T$ in $S$. Since $S$ contains no net, kite, or $K_{4}$, there is some vertex of $T$ of degree 2 in $S$. Let $T=\left\{a, b, c_{1}\right\}$ where $d_{S}\left(c_{1}\right)=2$. Let $c_{1}, c_{2}, \ldots, c_{t}$ be all the vertices of $S$ with $\{a, b\} \subseteq N_{S}\left(c_{i}\right)$. We have $\{a, b\}=N_{S}\left(c_{i}\right)$ as $S$ contains no kite. Since $S$ contains no $K_{4},\left\{c_{1}, \ldots, c_{t}\right\}$ is a stable set of $S$. Let $S^{\prime}$ be the graph that arises from $S$ by removing the edge $a b$ and the vertices $c_{1}, \ldots, c_{t}$. Since $S$ has no odd cycles of length at least 5 , there is no path joining $a$ and $b$ in $S^{\prime}$, but $S^{\prime}+a b$ is connected because $S$ is connected. Consequently, $S^{\prime}$ consists of two components $S_{1}^{\prime}$ and $S_{2}^{\prime}$ such that $a$ belongs to $S_{1}^{\prime}$ and $b$ to $S_{2}^{\prime}$. Let $S_{1}$ be the graph with a $t$-bloom $A=\left\{a ; a_{1}, \ldots, a_{t}\right\}$ obtained from $S_{1}^{\prime}$ by adding $t$ pendant vertices to $a$, and construct $S_{2}$ with a $t$-bloom $B$ analogously. Then $S=S_{1} \triangle_{A B} S_{2}$ clearly follows. Moreover, $S_{1}$ and $S_{2}$ satisfy (iv) because they are subgraphs of $S$. Therefore, as $S_{1}$ and $S_{2}$ are connected and have less edges than $S$, by induction hypothesis, $S_{1}, S_{2} \in \S$. This completes the proof of (iv) $\Rightarrow$ (v).

Let us now turn to the proof of $(\mathrm{v}) \Rightarrow(\mathrm{i})$. Assume that every component of $R$ belongs to $\&$. We will prove that $G=L(R)$ is balanced by induction on the number $n$ of edges of $R$. Without loss of generality we can assume that $R$ has no isolated vertices. If $n=1$, then $G=K_{1}$ is balanced. Let $n>1$ and assume that (i) holds when $R$ has less than $n$ edges. If $R$ is disconnected, each component $S$ of $R$ has less than $n$ edges and, by induction hypothesis, each $L(S)$ is balanced, hence $G=L(R)$ is balanced, as desired. If $R$ is connected, suppose, for the purpose of contradiction, that $G$ is not balanced; i.e., there exist $L$-cliques $E_{1}, \ldots, E_{r}$ and pairwise different edges $e_{1}, \ldots, e_{r}$ of $R$ such that $E_{i} \cap\left\{e_{1}, \ldots, e_{r}\right\}=\left\{e_{i}, e_{i+1}\right\}$ (from this point on, all subindices should be understood modulo $r$ ) for some odd $r \geq 3$.

Hence, $R$ is not bipartite and, since $R \in \delta$ by hypothesis, $R=R_{1} \triangle_{A B} R_{2}$ for $R_{1}, R_{2} \in \delta$ and $t$-blooms $A=\left\{a ; a_{1}, \ldots, a_{t}\right\}$ in $R_{1}$ and $B=\left\{b ; b_{1}, \ldots, b_{t}\right\}$ in $R_{2}$. Since $R_{1}$ and $R_{2}$ have less edges than $R$ then, by induction hypothesis, $L\left(R_{1}\right)$ and $L\left(R_{2}\right)$ are both balanced. If $S_{R}(a)$ is an $L$-clique of $R$, we will identify $S_{R}(a)$ with $S_{R_{1}}(a)$ and say that $S_{R}(a)$ is an L-clique of $R_{1}$. Similarly, if $S_{R}(b)$ is an $L$-clique of $R$, we identify $S_{R}(b)$ with $S_{R_{2}}(b)$ and will say that $S_{R}(b)$ is an $L$-clique of $R_{2}$. With this convention, the $L$-cliques of $R$ are the $L$-cliques of $R_{1}$ and $R_{2}$, plus the triads $T_{k}=\left\{a b, a c_{k}, b c_{k}\right\}$ for each $k=1, \ldots, t$, where $c_{k}$ is the vertex that results from contracting $a_{k}$ with $b_{k}$. If $r=3$, Theorem 2 implies that $G$ contains an induced pyramid, which means that $R$ contains net, kite, or $K_{4}$. Hence, by definition of $\triangle$, either $R_{1}$ or $R_{2}$ would contain net, kite or $K_{4}$, a contradiction to $L\left(R_{1}\right)$ and $L\left(R_{2}\right)$ balanced. Hence, we have $r \geq 5$ and suppose that at least one of $E_{1}, \ldots, E_{r}$ is an $L$-clique of $R_{1}$. Since $L\left(R_{1}\right)$ is balanced, not all of $E_{1}, \ldots, E_{r}$ are $L$-cliques of $R_{1}$. Therefore, there exists some $i \in\{1, \ldots, r\}$ such that $E_{i}$ is an $L$-clique of $R_{1}$, but $E_{i+1}$ is not. Since $E_{i} \cap E_{i+1} \neq \emptyset$, necessarily, $E_{i}=S_{R}(a)$. Similarly, there is some $j \in\{1, \ldots, r\}$ such that $E_{j}$ is an $L$-clique of $R_{1}$ and $E_{j-1}$ is not, and necessarily $E_{j}=S_{R}(a)$. Hence, every block of consecutive $L$-cliques of $R_{1}$ in the circular ordering $E_{1} E_{2} \ldots E_{r} E_{1}$ 
starts and ends with $S_{R}(a)$. Since $E_{1}, \ldots, E_{r}$ are $r$ pairwise different $L$-cliques of $R, S_{R}(a)$ is the only $L$-clique of $R_{1}$ that may belong to $E_{1}, \ldots, E_{r}$. Similarly, $S_{R}(b)$ is the only $L$-clique of $R_{2}$ that may belong to $E_{1}, \ldots, E_{r}$.

Since $r \geq 5$ and among $E_{1}, \ldots, E_{r}$ there are at most one $L$-clique of $R_{1}$ and at most one $L$-clique of $R_{2}$, there are two consecutive elements in the circular ordering $E_{1} E_{2} \ldots E_{k} E_{1}$ that are triads $T_{k}$ for some values of $k$. Without loss of generality, $E_{1}=T_{1}$ and $E_{2}=T_{2}$. Therefore, $e_{2} \in E_{1} \cap E_{2}=\{a b\}$. But then $e=a b$ belongs to each of $E_{1}, \ldots, E_{r}$, a contradiction. This contradiction arose from assuming that $G$ was not balanced. So, $G$ satisfies (i), as desired.

Theorem 9 implies another characterization of balanced graphs within line graphs that leads to a linear-time recognition algorithm for balanced line graphs.

Corollary 10. Consider a graph $R$ and its line graph $G=L(R)$. Let $U$ be the set of vertices of $R$ of degree 2 that belong to some triangle of $R$ and let $E^{\prime}$ be the set of edges of $R$ whose both endpoints are the two neighbors of some vertex of $U$. Then, $G$ is balanced if and only if $R-U$ is a bipartite graph and every edge of $R-U$ that belongs to $E^{\prime}$ is a bridge of $R-U$.

Proof. Suppose that $G$ is balanced. By Theorem 9(iv), $R$ contains no kite, net, or $K_{4}$. Thus, every triangle of $R$ has at least one vertex of degree 2 and, therefore, $R-U$ has no triangles. Since, in addition, $R$ has no odd cycles of length at least $5, R-U$ is bipartite. Moreover, any edge of $R-U$ in $E^{\prime}$ is a bridge. Suppose that, on the contrary, $a b$ belongs to some cycle $C$ of $R-U$. As $R-U$ is bipartite, $C=a b v_{1} \ldots v_{2 k} a$ for some $k \geq 1$. Since $a b \in E^{\prime}$ there exists some vertex $c \in R$ such that $N_{R}(c)=\{a, b\}$. But then $C^{\prime}=a c b v_{1} \ldots v_{2 k} a$ is a cycle of $R$ of length $2 k+3$ with $k \geq 1$, a contradiction since $R$ has no odd cycles of length at least 5.

Conversely, assume that $R-U$ is bipartite and every edge of $R-U$ that belongs to $E^{\prime}$ is a bridge of $R-U$. We will prove that statement (iv) of Theorem 9 holds. $R$ has no kite, net, or $K_{4}$ (otherwise, $R-U$ would contain a triangle in contradiction to $R-U$ bipartite). It only remains to prove that $R$ has no odd cycles of length at least 5 . Suppose, for the purpose of contradiction, that $R$ has a cycle $C=v_{1} v_{2} \ldots v_{r} v_{1}$ of odd length at least 5 . Let $w_{1}, w_{2}, \ldots, w_{s}$ be the sequence of vertices that arises from the sequence $v_{1}, v_{2}, \ldots, v_{r}$ by removing all the vertices that belong to $U$. Notice that, if $v_{i} \in U$, then $v_{i-1}$ and $v_{i+1}$ have degree at least 3 in $R$ and, therefore, $v_{i-1}$ and $v_{i+1}$ do not belong to $U$ and $v_{i-1} v_{i+1}$ is an edge of $R-U$. Therefore, $C^{\prime}=w_{1} w_{2} \ldots w_{s} w_{1}$ is a cycle of $R-U$. Since $C$ is an odd cycle and $R-U$ is bipartite, $C^{\prime} \neq C$. So, necessarily, there is at least one vertex of $C$ that belongs to $U$. Without loss of generality assume that $v_{2} \in U$. By construction, $w_{1}=v_{1}, w_{2}=v_{3}, v_{1} v_{3} \in E^{\prime}$, and $v_{1} v_{3}$ is an edge of the cycle $C^{\prime}$ in $R-U$. Therefore, $v_{1} v_{3}$ is an edge of $R-U$ that belongs to $E^{\prime}$ but is not a bridge of $R-U$, a contradiction. Hence, Theorem 9(iv) holds and, consequently, $G$ is balanced.

From Corollary 10, we deduce the following.

Corollary 11. It can be decided in linear time whether a given line graph $G$ is balanced.

Proof. Let $n$ and $m$ be the number of vertices and edges of $G$. A graph $R$ without isolated vertices such that $L(R)=G$ can be computed in $O(m+n)$ time [19,25]. Additionally, the neighborhoods of the vertices of $R$ can be easily sorted, consistently with some fixed total ordering of $V(R)$, in $O(n)$ time (see, e.g., [16, p. 115]). Notice that $O(n)$ time means linear time of $R$ because $R$ has $n$ edges and no isolated vertices. We now show that $U$ and $E^{\prime}$ defined as in Corollary 10 can also be computed in $O(n)$ time. Let $H$ be an auxiliary multigraph whose vertex set is $V(R)$ and having each of its edges labeled with a vertex of $R$ defined as follows: two vertices $v$ and $w$ of $H$ are joined by one (and exactly one) edge labeled with $x$ if and only if $N_{R}(x)=\{v, w\}$. Clearly, $H$ can be computed in $O(n)$ time and, as we did with $R$, we can sort the neighborhoods of $H$ (ignoring the edge labels), consistently with the total ordering of $V(R)$ used for the neighborhoods of $R$, also in $O(n)$ time. Now, as both $N_{R}(v)$ and $N_{H}(v)$ are sorted consistently for each $v \in V(R)$, we can find, in overall $O(n)$ time, the set $D$ of all triples $(v, w, x)$ that satisfy both that $w \in N_{R}(v) \cap N_{H}(v)$ and that there is an edge joining $v$ and $w$ labeled with $x$. Then, $U$ consists of all vertices $x$ such that there is some triple $(v, w, x) \in D$ and $E^{\prime}$ consists of all edges $v w$ such that there is some triple $(v, w, x) \in D$. This shows that indeed $U$ and $E^{\prime}$ can be computed in $O(n)$ time. Finally, we can also decide in $O(n)$ time whether $R-U$ is bipartite and whether the edges of $R-U$ that belong to $E^{\prime}$ are bridges of $R-U$, because the bridges of a graph can be determined by depth-first search in linear time [26].

Note. The sets $U$ and $E^{\prime}$ can also be computed in $O(m+n)$ time by enumerating all triangles of $R$ using the approach sketched in [16, p. 115], which leads to an alternative linear-time algorithm to decide the balancedness of G. Nevertheless, our procedure has the advantage that it takes only linear time of $R$ to decide the balancedness of $L(R)$ if $R$ is given as input.

We will now briefly comment on how the above results for line graphs naturally extend to line graphs of multigraphs. Since two vertices of the line graph $L(H)$ of a multigraph $H$ are adjacent if the corresponding edges in $H$ have at least one endpoint in common, two parallel edges of a multigraph $H$ are true twins in $L(H)$. This means that the line graph of the multigraph $H$ arises from the line graph of its underlying graph $\widehat{H}$ by adding true twins. As adding a true twin to a graph only duplicates one column of its clique-matrix, its balancedness is not affected. Therefore, $L(H)$ is balanced if and only if $L(\widehat{H})$ is balanced. Moreover, adding true twins does neither affect perfectness [21] nor being hereditary clique-Helly (the latter easily follows from Theorem 2 because no pyramid has true twins). Therefore, $L(H)$ is perfect and hereditary clique-Helly if and only if $L(\widehat{H})$ is so. As a consequence, Theorem 9 extends to line graphs of multigraphs as follows.

Theorem 12. Let $G$ be the line graph of a multigraph $H$. Then, the following assertions are equivalent:

(i) $G$ is balanced. 


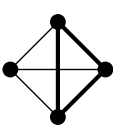

$\mathcal{A}_{1}$

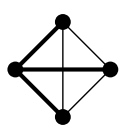

$\mathcal{A}_{2}$

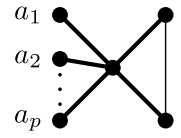

$\mathcal{A}_{3}$

Fig. 5. Multigraphs families $\mathcal{A}_{1}, \mathcal{A}_{2}$, and $\mathcal{A}_{3}$. Light lines represent single edges, whereas bold lines represent one or more parallel edges. Parameter $p$ varies over all positive integers and $a_{1}, a_{2}, \ldots, a_{p}$ are pairwise false twins.

(ii) $G$ is perfect and hereditary clique-Helly.

(iii) G has no odd holes and contains no induced 3-sun, 1-pyramid, or 3-pyramid.

(iv) $H$ has no odd cycles of length at least 5 and contains no net, kite, or $K_{4}$.

(v) Each component of the underlying graph of $H$ belongs to the class \& (as defined in the statement of Theorem 9).

Finally, also the linear-time recognition algorithm for balanced graphs within line graphs can be extended to line graphs of multigraphs. For that we need to introduce the following notion. A representative graph $\mathcal{R}(G)$ of $G$ is a graph that arises from $G$ by successively removing one vertex of some pair of true twins, as long as this is possible. It is easy to see that $\mathcal{R}(G)$ is unique up to isomorphisms. In [16], an algorithm is proposed that, given a graph $G$, computes $\mathcal{R}(G)$ in linear time.

Corollary 13. Given the line graph $G$ of a multigraph, it can be decided in linear time whether $G$ is balanced.

Proof. As $G=L(H)$ arises from $L(\widehat{H})$ by adding true twins, $\mathcal{R}(G)$ is also the representative graph of $L(\widehat{H})$. Thus, $\mathcal{R}(G)$ is an induced subgraph of $L(\widehat{H})$ and, in particular, $\mathcal{R}(G)$ is a line graph. In addition, as adding true twins does not affect balancedness, $G$ is balanced if and only if $\mathcal{R}(G)$ is balanced. We conclude that the algorithm for computing the representative graph in [16] reduces the problem of deciding the balancedness of the line graphs of multigraphs $G$ to that of deciding the balancedness of the line graphs $\mathcal{R}(G)$, which, as we have seen, is linear-time solvable.

\section{Balancedness of complements of line graphs of multigraphs}

We say that a multigraph $H$ is $\bar{L}$-balanced if the complement of its line graph is balanced. In this subsection, we will characterize those complements of line graphs of multigraphs that are balanced by determining which multigraphs are $\bar{L}$-balanced. As pairwise adjacent vertices in $\overline{L(H)}$ correspond to pairwise non-incident edges, i.e. matchings, in $H$, the cliquematrix of $\overline{L(H)}$ equals the maximal matching/edge-incidence matrix of $H$, which we call the matching-matrix. Consequently, $H$ is $\bar{L}$-balanced if and only if its matching-matrix is balanced.

\subsection{Families of $\bar{L}$-balanced multigraphs}

The main result of this subsection is Theorem 17 which establishes that certain multigraph families are $\bar{L}$-balanced. The proof of this theorem splits into two parts. The first part will follow from a sufficient condition for $\bar{L}$-balancedness (Lemma 15). For this purpose, we introduce three multigraph families $\mathcal{A}_{1}, \mathcal{A}_{2}$, and $\mathcal{A}_{3}$. In Fig. 5, a generic member of each of these families is shown, where light lines represent single edges, bold lines one or more parallel edges, $p$ is any positive integer, and $a_{1}, \ldots, a_{p}$ are pairwise false twins. A graph $G$ is called trivially perfect [11] if and only if $G$ is $\left\{P_{4}, C_{4}\right\}$-free. Our next lemma shows that the multigraph families $\mathcal{A}_{1}, \mathcal{A}_{2}$, and $\mathcal{A}_{3}$ arise naturally when characterizing those multigraphs $H$ such that $\overline{L(H)}$ is trivially perfect.

Lemma 14. For a graph $\mathrm{G}$ being the complement of the line graph of a multigraph $\mathrm{H}$, the following assertions are equivalent:

(i) $G$ is trivially perfect.

(ii) $H$ contains no $P_{5}, 2 P_{3}, P_{3} \cup C_{2}$, or $2 C_{2}$.

(iii) Some component of $H$ is contained in some member of $\mathcal{A}_{1}, \mathcal{A}_{2}$, or $\mathcal{A}_{3}$, and each of the remaining components of $H$ has at most one edge.

Proof. The equivalence between (i) and (ii) follows immediately from the definitions of trivially perfect graphs and line graphs of multigraphs. It is also clear, by simple inspection, that each of the members of the families $\mathcal{A}_{1}, \mathcal{A}_{2}$, and $\mathcal{A}_{3}$ contains no $P_{5}, 2 P_{3}, P_{3} \cup C_{2}$, or $2 C_{2}$. Therefore, the same holds also for any submultigraph of them, which proves that (iii) implies (ii). To complete the proof, we prove that (ii) implies (iii). Recall that $\hat{d}_{H}(v)$ denotes the degree of $v$ in the underlying graph $\widehat{H}$ and that a vertex $v$ of $H$ is pendant if and only if $\hat{d}_{H}(v)=1$.

Suppose that $H$ satisfies (ii) and let $S$ be any component of $H$. First assume that $S$ is a multitree and let $P=v_{1} v_{2} \ldots v_{t}$ be a longest path in $S$. Since $S$ contains no $P_{5}$, necessarily $t \leq 4, v_{1}$ and $v_{t}$ are pendant vertices, and each neighbor of $v_{2}, \ldots, v_{t-1}$ outside $P$ is a pendant vertex. If $t \leq 3, S$ is contained in some member of $\mathcal{A}_{3}$, as desired. So, let $t=4$. Since $S$ contains no $2 P_{3}, P_{3} \cup C_{2}$, or $2 C_{2}$, we can assume, by symmetry, that there is a single edge joining $v_{1}$ to $v_{2}$ and $\hat{d}_{S}\left(v_{2}\right)=2$. We conclude that $S$ is contained in some member of $\mathcal{A}_{3}$, as desired. So, from now on, let $S$ be not a multitree and let $\ell$ be the length of the longest cycle of $S$. Since $S$ contains no $P_{5}, \ell=3$ or $\ell=4$. 


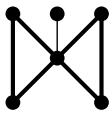

$\mathcal{B}_{1}$

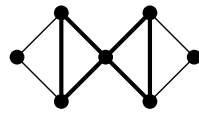

$\mathcal{B}_{2}$

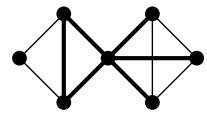

$\mathcal{B}_{3}$

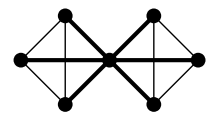

$\mathcal{B}_{4}$

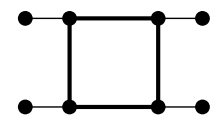

$\mathcal{B}_{5}$

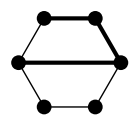

$\mathcal{B}_{6}$

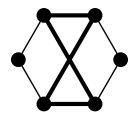

$\mathcal{B}_{7}$

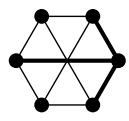

$\mathcal{B}_{8}$

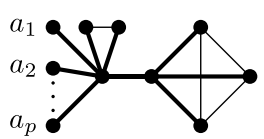

$\mathcal{B}_{9}$

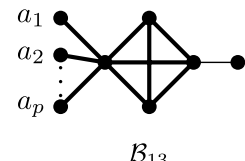

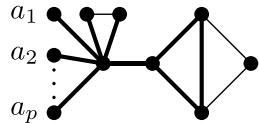

$\mathcal{B}_{10}$

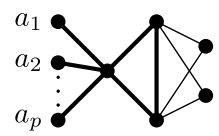

$\mathcal{B}_{14}$

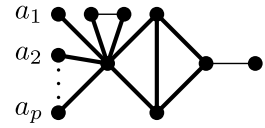

$\mathcal{B}_{11}$

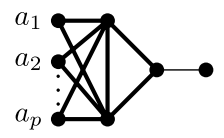

$\mathcal{B}_{15}$

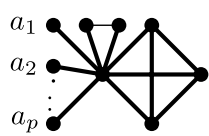

$\mathcal{B}_{12}$

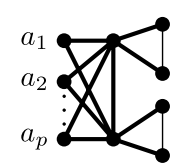

$\mathcal{B}_{16}$

Fig. 6. Multigraph families $\mathscr{B}_{1}, \mathscr{B}_{2}, \ldots, \mathscr{B}_{16}$. Light lines represent single edges, whereas bold lines represent one or more parallel edges. Parameter $p$ varies over the positive integers, and $a_{1}, a_{2}, \ldots, a_{p}$ are pairwise false twins.

If $\ell=3$, there is a triangle $T=v_{1} v_{2} v_{3} v_{1}$ in $S$. Since $S$ contains no $P_{5}$ or bipartite claw, at most one vertex of $T$ has some neighbor $v \in V(S) \backslash V(T)$ and each of these neighbors $v$ is a pendant vertex. Without loss of generality, we assume that $\hat{d}_{S}\left(v_{1}\right)=\hat{d}_{S}\left(v_{2}\right)=2$. If $\hat{d}_{S}\left(v_{3}\right)>3$ or $v_{3}$ is joined to some pendant vertex through two or more parallel edges, then there is a single edge joining $v_{1}$ to $v_{2}$ (because $S$ contains no $P_{3} \cup C_{2}$ or $2 C_{2}$ ) and $S$ is contained in some member of $\mathcal{A}_{3}$. If $\hat{d}_{S}\left(v_{3}\right) \leq 3$ and there are no two parallel edges joining $v_{3}$ to a pendant neighbor, then $S$ is contained in some member of $\mathcal{A}_{1}$.

Finally, suppose that $\ell=4$ and let $C$ be a 4-cycle of $S$. Since $C$ contains no $P_{5}$ or $2 C_{2}, V(S)=V(C)$ and $S$ has no two non-incident pairs of parallel edges. Therefore, $S$ is some member of $\mathcal{A}_{1}$ or $\mathcal{A}_{2}$.

We conclude that $H$ satisfies (iii), which completes the proof.

We say that two edges $e_{1}$ and $e_{2}$ of a multigraph $H$ are matching-separable if there is some maximal matching of $H$ that contains $e_{1}$ but not $e_{2}$, and vice versa. Notice that any two parallel edges are matching-separable. It is easy to see that, for each member of $\mathcal{A}_{1}, \mathcal{A}_{2}$, and $\mathscr{A}_{3}$, any two matching-separable edges are incident. Indeed, in each of the multigraphs represented in Fig. 5, the edges in bold are pairwise incident and each light edge is not matching-separable from any edge that is nonincident to it. Let $H$ be a multigraph. A submultigraph $F$ of $H$ is a fragment of $H$ if there is an embedding of $F$ in some of the multigraphs represented in Fig. 5 such that the edges of $F$ corresponding, under the embedding, to light edges in Fig. 5 are incident in $H$ to edges of $F$ only. The following is easy to verify by inspection.

Remark 1. If $F$ is a fragment of $H$, then any pair of edges of $F$ that are matching-separable in $H$ are incident.

In Fig. 6, we introduce multigraph families $\mathscr{B}_{1}, \mathscr{B}_{2}, \ldots, \mathscr{B}_{16}$ by presenting a generic member of each family: light lines represent single edges, bold lines one or more parallel edges, $p$ is any positive integer, and $a_{1}, \ldots, a_{p}$ are pairwise false twins. Notice, for instance, that for each member of $\mathscr{B}_{2}, \mathcal{B}_{3}$, and $\mathscr{B}_{4}$, its edge set can be partitioned into the edge sets of two fragments. Our next result shows that this condition is sufficient for $\bar{L}$-balancedness. Note that a $\{0,1\}$-matrix is balanced if and only if each of its submatrices is bicolorable [2], i.e., their columns can be partitioned into red and blue columns such that every row with two or more 1's contains a 1 of each color. If $H$ is a multigraph, $A$ is a submatrix of a matching-matrix of $H$, and $\mathcal{M}$ and $\varepsilon$ are the sets of maximal matchings and edges of $H$ corresponding to the rows and columns of $A$, then we say that a partition $\left\{\varepsilon_{1}, \varepsilon_{2}\right\}$ of $\mathscr{E}$ is a bicoloring of $A$ if for each $M \in \mathcal{M}$ either $|M \cap \mathscr{E}| \leq 1$ or $M$ intersects both $\mathcal{E}_{1}$ and $\varepsilon_{2}$.

Lemma 15. If the edge set of a multigraph $H$ can be partitioned into the edge sets of two fragments of $H$, then $H$ is $\bar{L}$-balanced.

Proof. Let $F_{1}$ and $F_{2}$ be two fragments of $H$ such that $\left\{E\left(F_{1}\right), E\left(F_{2}\right)\right\}$ is a partition of $E(H)$. Suppose, for the purpose of contradiction, that $\overline{L(H)}$ is not balanced. Then, any matching-matrix of $H$ has some submatrix $A$ that is an edge-vertex incidence matrix of an odd chordless cycle. Let $\mathcal{M}$ and $\&$ be the sets of maximal matchings and edges of $H$ corresponding to the rows and columns of the submatrix $A$, respectively.

We show that $\left\{E\left(F_{1}\right) \cap \mathscr{E}, E\left(F_{2}\right) \cap \mathscr{E}\right\}$ is a bicoloring of $A$. Since $A$ has no dominated rows, the edges in $\mathscr{E}$ are pairwise matching-separable in $H$. Hence, by Remark $1, E\left(F_{i}\right) \cap \mathscr{E}$ consists of pairwise incident edges, for each $i=1$, 2. So, if $M$ is any maximal matching of $H, M \cap \mathscr{E}$ consists of at most one element of $E\left(F_{1}\right)$ and at most one element of $E\left(F_{2}\right)$. 


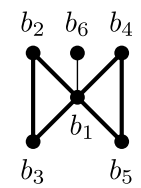

$H \in \mathcal{B}_{1}$

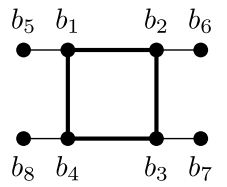

$H \in \mathcal{B}_{5}$

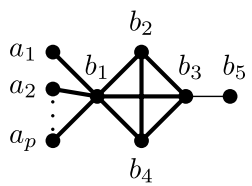

$H \in \mathcal{B}_{13}$

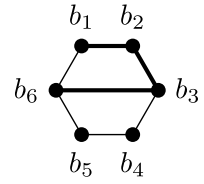

$H \in \mathcal{B}_{6}$

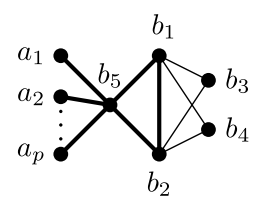

$H \in \mathcal{B}_{14}$

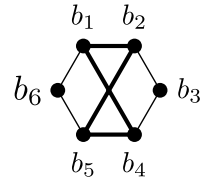

$H \in \mathcal{B}_{7}$

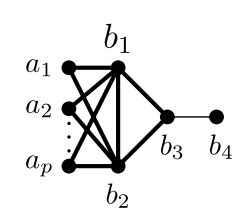

$H \in \mathcal{B}_{15}$

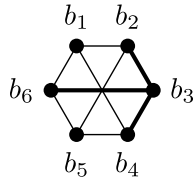

$H \in \mathcal{B}_{8}$

Fig. 7. Vertex labeling of the multigraph $H$ for the proof of Theorem 17. Light lines represent single edges, whereas bold lines represent one or more parallel edges. Parameter $p$ varies over the positive integers, and $a_{1}, a_{2}, \ldots, a_{p}$ are pairwise false twins.

Since $E(H)=E\left(F_{1}\right) \cup E\left(F_{2}\right)$ and, by construction, $|M \cap \mathcal{E}|=2$ for each $M \in \mathcal{M}$, it follows that, for each maximal matching $M \in \mathcal{M}, M \cap \mathcal{E}$ consists of one element of $E\left(F_{1}\right) \cap \mathcal{E}$ and one element of $E\left(F_{2}\right) \cap \mathcal{E}$. Hence, $\left\{E\left(F_{1}\right) \cap \mathcal{E}, E\left(F_{2}\right) \cap \mathcal{E}\right\}$ is a bicoloring of $A$, a contradiction to the choice of $A$. This proves that $H$ is $\bar{L}$-balanced.

Let $H$ be a multigraph. If $v$ is any vertex of $H, S_{H}(v)$ will denote the set of edges incident to $v$. If $u, v, w$ are three pairwise adjacent vertices of $H$, we denote by $T_{H}(u, v, w)$ the set of all edges of $H$ joining any two of the vertices $u, v$, and $w$.

We will make repeated use of the following lemma.

Lemma 16. Let $H$ be a not $\bar{L}$-balanced multigraph. Then, a matching-matrix of $H$ contains the edge-vertex incidence matrix of an odd chordless cycle as submatrix A. If $\mathcal{E}$ is the set of edges of $H$ corresponding to the columns of $A$ and $X$ a set of pairwise incident edges of $H$, there must be some maximal matching $M$ of $H$ with $|M \cap \mathcal{E}|=2$ and $M \cap \mathcal{E} \cap X=\emptyset$.

Proof. Let $\mathcal{M}$ be the set of maximal matchings of $H$ corresponding to the rows of the submatrix $A$. Since $A$ is an edge-vertex incidence matrix of an odd chordless cycle, $|M \cap \mathcal{E}|=2$ for each $M \in \mathcal{M}$. Since $X$ consists of pairwise incident edges of $X,|M \cap \mathcal{E} \cap X| \leq 1$ for every matching $M$ of $X$. As $|M \cap \mathscr{E}|=2$ for each $M \in \mathcal{M}$, it follows that $|(M \cap \mathcal{E}) \backslash X| \geq 1$ for each $M \in \mathcal{M}$. Since $A$ is not bicolorable, $\{X \cap \mathcal{E}, \mathcal{E} \backslash X\}$ is not a bicoloring of $A$ and, necessarily, there is some $M \in \mathcal{M}$ such that $|M \cap X \cap \mathcal{E}|=0$.

Theorem 17. The families $\mathscr{B}_{1}, \mathscr{B}_{2}, \ldots, \mathscr{B}_{16}$ are $\bar{L}$-balanced.

Proof. It follows, by direct application of Lemma 15 , that the families $\mathcal{B}_{2}, \mathcal{B}_{3}, \mathcal{B}_{4}, \mathcal{B}_{9}, \mathcal{B}_{10}, \mathcal{B}_{11}, \mathscr{B}_{12}$, and $\mathscr{B}_{16}$ are $\bar{L}$-balanced; i.e., each of its members is $\bar{L}$-balanced. In order to finish the proof, we shall verify the $\bar{L}$-balancedness for each of the remaining families $\mathcal{B}_{1}, \mathcal{B}_{5}, \mathcal{B}_{6}, \mathcal{B}_{7}, \mathscr{B}_{8}, \mathcal{B}_{13}, \mathscr{B}_{14}$, and $\mathscr{B}_{15}$ displayed in Fig. 6 . For that, we verify the assertion for each family $\mathscr{B}_{i}$ in a separate claim and suppose, for the purpose of contradiction, the following: $\operatorname{consider}$ a not $\bar{L}$-balanced $H \in \mathscr{B}_{i}$, label its vertices as in Fig. 7 and let $A$ and $\mathcal{E}$ be as in Lemma 16.

Claim 1 (The Family $B_{1}$ is $\bar{L}$-balanced). We have $b_{1} b_{6} \in \mathcal{E}:$ By Lemma 16 applied to $X=T_{H}\left(b_{1}, b_{2}, b_{3}\right)$, there is some maximal matching $M$ of $H$ such that $|M \cap \mathcal{E}|=2$ but $M \cap \& \cap X=\emptyset$. Necessarily, $b_{1} b_{6} \in M \cap \varepsilon$. By Lemma 16 applied to $X=S_{H}\left(b_{1}\right)$, there is some maximal matching $M$ of $H$ such that $|M \cap \mathscr{E}|=2$ but $M \cap \varepsilon \cap X=\emptyset$. Necessarily, $M \cap \varepsilon$ consists of one edge joining $b_{2}$ to $b_{3}$ and one edge joining $b_{4}$ to $b_{5}$ and, by the maximality of $M, b_{1} b_{6} \in M$. From $b_{1} b_{6} \in \&$, we conclude that $b_{1} b_{6} \in M \cap \mathcal{E} \cap X$, which contradicts $M \cap \mathcal{E} \cap X=\emptyset$. Hence, any member of $\mathcal{B}_{1}$ is $\bar{L}$-balanced.

Claim 2 (The Family $\mathscr{B}_{5}$ is $\bar{L}$-balanced). By Lemma 16 applied to $X=S_{H}\left(b_{2}\right)$, there is a maximal matching $M$ of $H$ such that $|M \cap \mathcal{E}|=2$ but $M \cap \mathcal{E} \cap X=\emptyset$. So, necessarily, $M \cap \&$ contains at least one of $b_{1} b_{5}$ and $b_{3} b_{7}$. Symmetrically, $M \cap \&$ contains at least one of $b_{2} b_{6}$ and $b_{4} b_{8}$. So, we assume, without loss of generality, that $b_{1} b_{5}, b_{2} b_{6} \in \mathcal{E}$.

We show that $b_{1} b_{5}, b_{2} b_{6} \in \mathcal{E}$ implies $b_{3} b_{7}, b_{4} b_{8} \in \mathcal{E}$. By Lemma 16 applied to $X=S_{H}\left(b_{1}\right)$, there is some maximal matching $M$ of $H$ such that $|M \cap \mathscr{E}|=2$ but $M \cap \& \cap X=\emptyset$. As $b_{1} b_{5} \in \mathscr{E} \cap X$, it follows that $b_{1} b_{5} \notin M$. Thus, by maximality, M contains an edge joining $b_{1}$ to either $b_{2}$ or $b_{4}$. Then, as $M \cap \&$ consists of two non-incident edges and is disjoint from $S_{H}\left(b_{1}\right)$, necessarily $b_{3} b_{7} \in M \cap \mathcal{E}$ and, in particular, $b_{3} b_{7} \in \mathcal{E}$. Symmetrically, $b_{4} b_{8} \in \mathcal{E}$.

Let $R=\left(S_{H}\left(b_{2}\right) \cup\left\{b_{4} b_{8}\right\}\right) \cap \mathcal{E}$ and $B=\mathcal{E} \backslash R$. We show that the partition $\{R, B\}$ of $\mathcal{E}$ is a bicoloring of $A$. Let $\mathcal{M}$ be the set of maximal matchings of $H$ corresponding to the rows of $A$ and let $M \in \mathcal{M}$. As $A$ is an edge-vertex incidence matrix of an odd chordless cycle, $|M \cap \mathbb{E}|=2$. Suppose, for the purpose of contradiction, that $M \cap R=\emptyset$. This means that $M \cap \&$ is disjoint from $S_{H}\left(b_{2}\right) \cup\left\{b_{4} b_{8}\right\}$. So, since $|M \cap \mathcal{E}|=2, M \cap \mathcal{E}$ consists of one edge incident to $b_{1}$ and one edge incident to $b_{3}$ but none of them incident to $b_{2}$ and, by maximality, $b_{2} b_{6} \in M$. Consequently, $b_{2} b_{6} \in M \cap R$, a contradiction. 
Suppose now that $M \cap B=\emptyset$. This means that $M \cap \mathcal{E}$ consists of two edges contained in $S_{H}\left(b_{2}\right) \cup\left\{b_{4} b_{8}\right\}$. Since $M$ is a matching, $M \cap \mathcal{E}$ consists of $b_{4} b_{8}$ and one edge incident to $b_{2}$. Then, the maximality of $M$ implies $M \cap\left\{b_{1} b_{5}, b_{3} b_{7}\right\} \neq \emptyset$ and $(M \cap B) \cap\left\{b_{1} b_{5}, b_{3} b_{7}\right\} \neq \emptyset$, a contradiction.

So, we have proved that for each $M \in \mathcal{M}, M \cap R \neq \emptyset$ and $M \cap B \neq \emptyset$, which verifies that $\{R, B\}$ is a bicoloring of $A$, contradicting the choice of A. Hence, any member of $\mathscr{B}_{5}$ is $\bar{L}$-balanced.

Claim 3 (The Family $\mathscr{B}_{6}$ is $\bar{L}$-balanced). We have $b_{4} b_{5} \in \mathcal{E}$ : Otherwise, by Lemma 16 applied to $X=S_{H}\left(b_{3}\right)$, there is some maximal matching $M$ of $H$ such that $|M \cap \mathcal{E}|=2$ but $M \cap \mathcal{E} \cap X=\emptyset$. Necessarily, $M \cap \mathcal{E}$ consists of the edge $b_{5} b_{6}$ and an edge joining $b_{1}$ to $b_{2}$. In particular, $b_{5} b_{6} \in \mathcal{E}$. Similarly, by Lemma 16 applied to $X=S_{H}\left(b_{6}\right)$, there is some maximal matching $M$ of $H$ with $|M \cap \mathscr{E}|=2$ and $M \cap \mathscr{E} \cap X=\emptyset$. So, $M \cap \mathscr{E}$ consists of $b_{3} b_{4}$ and an edge joining $b_{1}$ to $b_{2}$. Hence, the maximality of $M$ implies that $b_{5} b_{6} \in M$ and from $b_{5} b_{6} \in \mathcal{E}$ follows $b_{5} b_{6} \in M \cap \& \cap X$, contradicting $M \cap \& \cap X=\emptyset$.

Moreover, no edge joining $b_{3}$ to $b_{6}$ belongs to $\mathcal{E}$ : Otherwise, if there is some edge $e \in \mathcal{E}$ joining $b_{3}$ to $b_{6}$, let $\mathcal{M}$ be the set of maximal matchings of $H$ corresponding to the rows of $A$. As $A$ is an edge-vertex incidence matrix of an odd chordless cycle, there are two different maximal matchings $M, M^{\prime} \in \mathcal{M}$ such that $|M \cap \mathcal{E}|=\left|M^{\prime} \cap \mathcal{E}\right|=2$ and e $\in$ M, $M^{\prime}$. Since every maximal matching of $H$ containing e also contains $b_{4} b_{5}$, we conclude that $M \cap \mathscr{E}=M^{\prime} \cap \mathcal{E}=\left\{e, b_{4} b_{5}\right\}$. This means that rows and columns of A corresponding to $M, M^{\prime}$ and e, $b_{4} b_{5}$ determine $a 2 \times 2$ submatrix of A full of 1's, which contradicts the choice of $A$.

We also have $b_{5} b_{6} \in \mathcal{E}$ : Otherwise, if $b_{5} b_{6} \notin \&$, by Lemma 16 applied to $X=S_{H}\left(b_{4}\right)$, there is some maximal matching $M$ of $H$ such that $|M \cap \&|=2$ and $M \cap \& \cap X=\emptyset$. As $b_{5} b_{6} \notin \varepsilon, M \cap \&$ consists of $b_{1} b_{6}$ and an edge joining $b_{2}$ to $b_{3}$ and the maximality of $M$ implies that $b_{4} b_{5} \in M$. Thus, since $b_{4} b_{5} \in \mathcal{E}$, it follows that $b_{4} b_{5} \in M \cap \mathcal{E} \cap X$, which contradicts $M \cap \mathcal{E} \cap X=\emptyset$.

We further have $b_{3} b_{4}, b_{1} b_{6} \in \mathcal{E}$. By Lemma 16 applied to $X=S_{H}\left(b_{5}\right)$, there is some maximal matching $M$ of $H$ such that $|M \cap \mathcal{E}|=2$ and $M \cap \mathcal{E} \cap X=\emptyset$. Similar argumentations as above show $b_{3} b_{4} \in M \cap \mathcal{E}$. If $b_{1} b_{6} \notin M \cap \mathcal{E}$, then $M \cap \mathcal{E}$ consists of $b_{3} b_{4}$ and an edge joining $b_{1} b_{2}$ and, by maximality, $b_{5} b_{6} \in M$. But then $b_{5} b_{6} \in \mathcal{E}$ implies $b_{5} b_{6} \in M \cap \mathcal{E} \cap X$, a contradiction. Hence, $b_{1} b_{6} \in M \cap \&$ and, in particular $b_{3} b_{4}, b_{1} b_{6} \in \mathcal{E}$, indeed follows.

Let $R=\left(S_{H}\left(b_{3}\right) \cup\left\{b_{4} b_{5}\right\}\right) \cap \mathcal{E}$ and $B=\& \backslash R$. Analogously to the proof for $\mathscr{B}_{5}$, one can show that the partition $\{R, B\}$ of $\&$ is a bicoloring of $A$, which contradicts the choice of $A$. Hence, any member of $\mathcal{B}_{6}$ is $\bar{L}$-balanced.

Claim 4 (The Family $\mathcal{B}_{7}$ is $\bar{L}$-balanced). By Lemma 16 applied to $X=S_{H}\left(b_{2}\right)$, there is some maximal matching $M$ such that $|M \cap \mathcal{E}|=2$ and $M \cap \mathcal{E} \cap X=\emptyset$. Since $M \cap \mathcal{E}$ is a matching of size 2 disjoint from $X$, at least one of $b_{1} b_{6}$ and $b_{5} b_{6}$ belongs to $M \cap \mathcal{E}$ and, in particular, to $\mathcal{E}$. By symmetry, assume that $b_{1} b_{6} \in \mathcal{E}$. We next show that this implies $b_{5} b_{6} \in \mathcal{E}$ and, moreover, $b_{2} b_{3} \in \mathcal{E}$ or $b_{3} b_{4} \in \&$. By Lemma 16 applied to $X=S_{H}\left(b_{1}\right)$, there is some maximal matching $M$ of $H$ such that $|M \cap \&|=2$ and $M \cap \mathcal{E} \cap X=\emptyset$. We have $b_{5} b_{6} \in M \cap \mathcal{E}$ : Otherwise $M \cap \&$ consists either of $b_{2} b_{3}$ and one edge joining $b_{4}$ to $b_{5}$, or of $b_{3} b_{4}$ and one edge joining $b_{2}$ to $b_{5}$. In either case, the maximality of $M$ implies that $b_{1} b_{6} \in M$ and, from $b_{1} b_{6} \in \&$ follows $b_{1} b_{6} \in M \cap \& \cap X$, which contradicts $M \cap \mathcal{E} \cap X=\emptyset$. As $M \cap \mathcal{E}$ is a matching of size 2 , disjoint from $X$, and containing $b_{5} b_{6}$, necessarily $b_{2} b_{3} \in M \cap \&$ or $b_{3} b_{4} \in \mathcal{M} \cap \mathcal{E}$. This verifies $b_{5} b_{6} \in \mathcal{E}$ and we may assume further $b_{2} b_{3}, b_{5} b_{6} \in \mathcal{E}$.

Reasoning as in the previous paragraph, $b_{2} b_{3} \in \mathcal{E}$ implies $b_{3} b_{4} \in \mathcal{E}$. We conclude that $\varepsilon_{1}=\left\{b_{1} b_{6}, b_{2} b_{3}, b_{3} b_{4}, b_{5} b_{6}\right\}$ is contained in $\mathcal{E}$. Let $R=\left(S_{H}\left(b_{2}\right) \cup\left\{b_{5} b_{6}\right\}\right) \cap \mathcal{E}, B=\mathcal{E} \backslash R$, and $M$ be a maximal matching of $H$ corresponding to a row of $A$. By construction, $|M \cap \mathcal{E}|=2$. If $\left|M \cap \mathcal{E}_{1}\right|=2$, necessarily $M$ has an edge in $R$ and an edge in $B$. Notice that if $\left|M \cap \mathcal{E}_{1}\right| \neq 2$, necessarily $M \cap \varepsilon_{1}=\varnothing$ and, since $M \cap \varepsilon$ is a matching of size 2, $M$ also has one edge in $R$ and one edge in $B$. This shows that $\{R, B\}$ is a bicoloring of $A$, which contradicts the choice of $A$. Hence, any member of $\mathcal{B}_{7}$ is $\bar{L}$-balanced.

Claim 5 (The Family $\mathscr{B}_{8}$ is $\bar{L}$-balanced). By Lemma 16 applied to $X=S_{H}\left(b_{3}\right)$, there is some maximal matching $M$ of $H$ such that $|M \cap \mathcal{E}|=2$ and $M \cap \mathcal{E} \cap X=\emptyset$. By symmetry, assume $M \cap \mathcal{E}=\left\{b_{1} b_{2}, b_{4} b_{5}\right\}$ and, in particular, $b_{1} b_{2}, b_{4} b_{5} \in \mathcal{E}$. No edge joining $b_{3}$ to $b_{6}$ belongs to $\varepsilon$ : Otherwise, if there is some edge $e \in \mathcal{E}$ joining $b_{3}$ to $b_{6}$, then there are two different maximal matchings $M$ and $M^{\prime}$ of $H$ such that $e \in M, M^{\prime}$ and $|M \cap \mathcal{E}|=\left|M^{\prime} \cap \mathcal{E}\right|=2$ because $A$ is an edge-vertex incidence matrix of an odd chordless cycle. But $\left\{e, b_{1} b_{2}, b_{4} b_{5}\right\}$ and $\left\{e, b_{1} b_{4}, b_{2} b_{5}\right\}$ are the only maximal matchings of $H$ containing $e$ and $\left|\left\{e, b_{1} b_{2}, b_{4} b_{5}\right\} \cap \mathcal{E}\right|=3$, a contradiction.

We now show that this implies $b_{1} b_{6} \in \mathcal{E}$ or $b_{5} b_{6} \in \mathcal{E}$. By Lemma 16 applied to $X=S_{H}\left(b_{2}\right)$, there is some maximal matching $M$ of $H$ such that $|M \cap \&|=2$ and $M \cap \& \cap X=\emptyset$. Necessarily, $M \cap \&$ consists of one edge incident to $b_{4}$ and one edge incident to $b_{6}$ and, since no edge joining $b_{3}$ to $b_{6}$ belongs to $\mathcal{E}$, it follows that $M \cap \&$ contains $b_{1} b_{6}$ or $b_{5} b_{6}$. In particular, $b_{1} b_{6} \in \mathcal{E}$ or $b_{5} b_{6} \in \mathcal{E}$. By symmetry, assume $b_{1} b_{6} \in \mathcal{E}$.

We further have $b_{5} b_{6} \in \mathcal{E}:$ By Lemma 16 applied to $X=S_{H}\left(b_{1}\right)$, there is some maximal matching $M$ of $H$ such that $|M \cap \&|=2$ and $M \cap \mathcal{E} \cap X=\emptyset$. If $b_{5} b_{6} \notin \mathcal{E}$, in particular, $b_{5} b_{6} \notin M \cap \mathcal{E}$. As no edge joining $b_{3}$ to $b_{6}$ belongs to $\mathcal{E}, M \cap \&$ consists either of the edge $b_{2} b_{5}$ and an edge joining $b_{3}$ to $b_{4}$, or of the edge $b_{4} b_{5}$ and an edge joining $b_{2}$ to $b_{3}$. In either case, the maximality of $M$ implies that $b_{1} b_{6} \in M$ and, since $b_{1} b_{6} \in \mathcal{E}$, it follows that $b_{1} b_{6} \in M \cap \mathcal{E} \cap X$, contradicting $M \cap \mathcal{E} \cap X=\emptyset$.

As $b_{1} b_{2}, b_{4} b_{5} \in \mathcal{E}$ implies $b_{1} b_{6}, b_{5} b_{6} \in \mathcal{E}$, by symmetry, $b_{1} b_{2}, b_{5} b_{6} \in \mathcal{E}$ shows $b_{1} b_{4} \in \mathcal{E}$. Similarly, from $b_{1} b_{6}, b_{4} b_{5} \in \mathcal{E}$ follows $b_{2} b_{5} \in \mathcal{E}$. We infer $S_{H}\left(b_{1}\right) \cup S_{H}\left(b_{5}\right) \subseteq \mathcal{E}$. Let $R=S_{H}\left(b_{1}\right)$ and $B=\mathcal{E} \backslash R$. Any maximal matching $M$ of $H$ contains one edge incident to $b_{1}$, one edge incident to $b_{3}$, and one edge incident to $b_{5}$. As $S_{H}\left(b_{1}\right)=R$ and $S_{H}\left(b_{5}\right) \subseteq B, M$ contains one edge from $R$ and at least one edge from $B$. Thus, $\{R, B\}$ is a bicoloring of $A$, contradicting the choice of $A$. Hence, any member of $B_{8}$ is $\bar{L}$-balanced.

Claim 6 (The Family $\mathcal{B}_{13}$ is $\bar{L}$-balanced). By Lemma 16 applied to $X=S_{H}\left(b_{1}\right)$, there is some maximal matching $M$ of $H$ such that $|M \cap \mathcal{E}|=2$ but $M \cap \mathcal{E} \cap X=\emptyset$. Necessarily $b_{3} b_{5} \in M \cap \mathcal{E}$ and, in particular, $b_{3} b_{5} \in \mathcal{E}$. 
By Lemma 16 applied to $X=S_{H}\left(b_{3}\right)$, there is some maximal matching of $M$ such that $|M \cap \&|=2$ but $M \cap \mathcal{E} \cap X=\emptyset$. Necessarily, some edge joining $b_{2}$ to $b_{4}$ belongs to $M \cap \mathcal{E}$ and, by the maximality of $M, b_{3} b_{5} \in M$. Hence, as $b_{3} b_{5} \in \mathcal{E}$, we conclude that $b_{3} b_{5} \in M \cap \mathcal{E} \cap X$, which contradicts $M \cap \mathcal{E} \cap X=\emptyset$. Hence, any member of $\mathscr{B}_{13}$ is $\bar{L}$-balanced.

Claim 7 (The Family $\mathcal{B}_{14}$ is $\bar{L}$-Balanced). By Lemma 16 applied to $X=S_{H}\left(b_{1}\right)$, there is some maximal matching $M$ of $H$ such that $|M \cap \mathcal{E}|=2$ but $M \cap \mathcal{E} \cap X=\emptyset$. Necessarily, $b_{2} b_{3} \in M \cap \mathcal{E}$ or $b_{2} b_{4} \in M \cap \mathcal{E}$. By symmetry, assume $b_{2} b_{3} \in M \cap \&$. Then, the maximality of $M$ implies $b_{1} b_{4} \in M$ and $b_{1} b_{4} \notin \varepsilon$ (otherwise, $b_{1} b_{4}$ would belong to $M \cap \& \cap X$ ).

By Lemma 16 applied to $X=S_{H}\left(b_{3}\right)$, there is some maximal matching $M$ such that $|M \cap \mathscr{E}|=2$ but $M \cap \& \cap X=\emptyset$. From $b_{1} b_{4} \notin \&$ follows $b_{1} b_{3} \in M \cap \mathcal{E}$. By maximality, $b_{2} b_{4} \in M$ and $b_{2} b_{4} \notin \&$ (otherwise, $b_{2} b_{4} \in M \cap \& \cap X$.)

By Lemma 16 applied to $X=T_{H}\left(b_{1}, b_{2}, b_{3}\right)$, there is some maximal matching $M$ of $H$ such that $|M \cap \varepsilon|=2$ but $M \cap \varepsilon \cap X=\emptyset$. Necessarily, $M \cap \mathcal{E}$ contains an edge incident to $b_{4}$ and, in particular, $b_{1} b_{4} \in \mathcal{E}$ or $b_{2} b_{4} \in \mathcal{E}$, which contradicts the conclusion of the preceding two paragraphs.

Hence, any member of $\mathscr{B}_{14}$ is $\bar{L}$-balanced.

Claim 8 (The Family $B_{15}$ is $\bar{L}$-Balanced). By Lemma 16 applied to $R=S_{H}\left(b_{1}\right)$, there is some maximal matching $M$ of $H$ such that $|M \cap \mathcal{E}|=2$ but $M \cap \mathcal{E} \cap X=\emptyset$. Necessarily $b_{3} b_{4} \in M \cap \mathcal{E}$ and, in particular, $b_{3} b_{4} \in \mathcal{E}$.

By Lemma 16 applied to $X=S_{H}\left(b_{3}\right)$, there is some maximal matching $M$ of $H$ such that $|M \cap \varepsilon|=2$ but $M \cap \mathcal{E} \cap X=\emptyset$. Necessarily, $M \cap \mathcal{E}$ consists one edge incident to $b_{1}$ and one edge incident to $b_{2}$, but none of them incident to $b_{3}$. Hence, by the maximality of $M, b_{3} b_{4} \in M$ and, as we proved that $b_{3} b_{4} \in \mathcal{E}$, we conclude that $b_{3} b_{4} \in M \cap \varepsilon \cap X$, which contradicts $M \cap \mathcal{E} \cap X=\emptyset$. Hence, any member of $\mathcal{B}_{15}$ is $\bar{L}$-balanced.

Hence, any member of each of the families $\mathscr{B}_{1}, \ldots, \mathcal{B}_{16}$ is $\bar{L}$-balanced.

\subsection{Characterizing balanced complements of line graphs}

In this subsection, we characterize those complements of line graphs of multigraphs that are balanced, including a characterization by minimal forbidden induced subgraphs.

Theorem 18. Let $G$ be the complement of the line graph of a multigraph $H$. Then, the following assertions are equivalent:

(i) $G$ is balanced.

(ii) A clique-matrix of $G$ has no edge-vertex incidence matrix of $C_{3}, C_{5}$, or $C_{7}$ as a submatrix.

(iii) $G$ contains no induced 3-sun, 2-pyramid, 3-pyramid, $C_{5}, \overline{C_{7}}, U_{7}$, or $V_{7}$.

(iv) $H$ contains no bipartite claw, $P_{5} \cup P_{3}, P_{5} \cup C_{2}, 3 P_{3}, 2 P_{3} \cup C_{2}, P_{3} \cup 2 C_{2}, 3 C_{2}, C_{5}, C_{7}$, 6-pan, braid, 1-braid, or 2-braid.

(v) One of the following conditions holds:

(a) Each component of $H$ has at most one edge.

(b) $H$ has exactly one component with more than one edge, which is contained in a member of $\mathscr{B}_{1}, \mathscr{B}_{2}, \ldots$, or $\mathscr{B}_{16}$.

(c) H has exactly two components with more than one edge each, each of which is contained in a member of $\mathcal{A}_{1}, \mathcal{A}_{2}$, or $\mathcal{A}_{3}$.

Proof. The implication (i) $\Rightarrow$ (ii) follows by definition. The implication (ii) $\Rightarrow$ (iii) follows from the fact that a clique-matrix of each of 3-sun, 2-pyramid, 3-pyramid, $C_{5}, \overline{C_{7}}, U_{7}$, and $V_{7}$ has an edge-vertex incidence matrix of $C_{3}, C_{5}$, or $C_{7}$ as a submatrix. The implication (iii) $\Rightarrow$ (iv) follows by definition of the line graph of a multigraph.

The implication ( $\mathrm{v}) \Rightarrow$ (i) can be proved as follows. If $(\mathrm{v})$.(a) holds, then $G=\overline{L(H)}$ is a clique and, in particular, $G$ is balanced. So, assume that (v).(b) or (v).(c) holds. Without loss of generality, $H$ has no isolated vertices. Moreover, we can also assume that $H$ has no component with only one edge because removing these components from $H$ amounts to removing the universal vertices from $\overline{L(H)}$, which does not affect the balancedness of $\overline{L(H)}$ (because each universal vertex corresponds to a column full of 1's in the clique-matrix). Therefore, we can assume that $H$ is contained in a member of $\mathcal{B}_{1}, \mathscr{B}_{2}, \ldots$, or $\mathcal{B}_{16}$ or $H$ has two components, each of which is contained in a member of $\mathscr{A}_{1}, \mathcal{A}_{2}$, or $\mathcal{A}_{3}$. If the former holds, $\overline{L(H)}$ is balanced by Theorem 17, if the latter holds, $\overline{L(H)}$ is balanced by Lemma 15 . This concludes the proof of $(\mathrm{v}) \Rightarrow(\mathrm{i})$.

The rest of the proof is devoted to showing that (iv) $\Rightarrow(\mathrm{v})$. In order to do so, assume that $H$ satisfies (iv). Suppose first that $H$ has two or more components with two or more edges each. Since $H$ contains no $3 P_{3}, 2 P_{3} \cup C_{2}, P_{3} \cup 2 C_{2}$, or $3 C_{2}, H$ has exactly two components $S_{1}$ and $S_{2}$ with at least two edges each. In particular, $S_{2}$ contains $P_{3}$ or $C_{2}$, which means that $S_{1}$ contains no $P_{5}, 2 P_{3}, P_{3} \cup C_{2}$, or $2 C_{2}$ and, by Lemma $14, S_{1}$ is contained in some member of $\mathscr{A}_{1}, \mathcal{A}_{2}$, or $\mathcal{A}_{3}$. By symmetry, $S_{2}$ is also contained in some member of $\mathcal{A}_{1}, \mathcal{A}_{2}$, or $\mathcal{A}_{3}$. This proves that if $H$ has at least two components with two or more edges each, (c) holds. If each component of $H$ has at most one edge, (a) holds. Therefore, we assume that $H$ has exactly one component $S$ having at least two edges. We will prove that $S$ is contained in some member of $\mathscr{B}_{1}, \mathscr{B}_{2}, \ldots, \mathscr{B}_{16}$ and, consequently, (b) holds, concluding the proof of the theorem.

We split the proof into four main cases. In the first case $S$ is a multitree. In the other cases, we assume that $S$ is not a multitree and we let $\ell$ be the length of the longest cycle in $S$. Since $S$ contains no $C_{5}, C_{7}$, or $P_{5} \cup P_{3}$, necessarily $\ell=3$, 4 , or 6 .

Along this proof, we adopt the following convention: Given any two adjacent vertices $u$ and $v$ of $S$, we will say that $u v$ is a simple edge if there is exactly one edge joining $u$ to $v$; otherwise, we say that $u v$ is a multiple edge. Recall that we say that a vertex $v$ of $S$ is pendant if and only if $\hat{d}(v)=1$ (where $\hat{d}_{S}(v)$ denotes the degree of $v$ in the underlying graph $\widehat{S}$ ). 
Case $1 . S$ is a multitree. Let $P=v_{1} v_{2} \ldots v_{t}$ be a path of $S$ of maximum length. By maximality of $P$ and since $S$ contains no bipartite claw, the endpoints of $P$ are pendant vertices and the neighbors of $v_{2}, \ldots, v_{t-1}$ outside $P$ are pendant vertices. Moreover, $2 \leq t \leq 7$ because $S$ contains no $P_{5} \cup P_{3}$.

1.a $t \leq 4$. Then, $S$ is contained in some member of $\mathcal{B}_{15}$.

1.b $t=5$. If $\hat{d}_{S}\left(v_{3}\right) \leq 3$ and any edge joining $v_{3}$ to a pendant neighbor is simple, then $S$ is contained in some member of $\mathscr{B}_{15}$. If $\hat{d}_{S}\left(v_{3}\right)>3$ or there is a multiple edge joining $v_{3}$ to a pendant neighbor, then either $\hat{d}_{S}\left(v_{2}\right)=2$ and $v_{1} v_{2}$ is simple, or $\hat{d}_{S}\left(v_{4}\right)=2$ and $v_{4} v_{5}$ is simple (otherwise $S$ would contain $3 P_{3}, 2 P_{3} \cup C_{2}, P_{3} \cup 2 C_{2}$, or $3 C_{2}$ ). In either case, $S$ is contained in some member of $\mathcal{B}_{16}$.

1.c $t=6$. If $\hat{d}_{S}\left(v_{2}\right)=\hat{d}_{S}\left(v_{5}\right)=2$ and $v_{1} v_{2}$ and $v_{5} v_{6}$ are simple, then $S$ is contained in some member of $\mathscr{B}_{16}$. If, say, $\hat{d}_{S}\left(v_{2}\right)>2$ or $v_{1} v_{2}$ is multiple, then $\hat{d}_{S}\left(v_{3}\right)=2$ follows since $S$ contains no $P_{5} \cup P_{3}$ and no $P_{5} \cup C_{2}$. In addition, $\hat{d}_{S}\left(v_{5}\right)=2$ and $v_{5} v_{6}$ is simple, because $S$ contains no braid, 1-braid, or 2-braid. Thus, $S$ is also contained in some member of $B_{16}$.

1.d $t=7$. Since $S$ contains no $P_{5} \cup P_{3}$ and no $P_{5} \cup C_{2}, \hat{d}_{S}\left(v_{2}\right)=\hat{d}_{S}\left(v_{4}\right)=\hat{d}_{S}\left(v_{6}\right)=2$ and the edges $v_{1} v_{2}$ and $v_{6} v_{7}$ are simple. Therefore, $S$ is contained in some member of $\mathscr{B}_{16}$.

Case 2. S has a longest cycle of length $\ell=3$. In each subcase, we assume that the previous subcases do not hold.

2.a There is some triangle $T$ such that all its vertices have some neighbor outside $T$. Let $T=v_{1} v_{2} v_{3} v_{1}$ be such a triangle in $S$. By hypothesis, $S$ has neither a 4-cycle nor a bipartite claw. Therefore, for each $i=1,2$, 3, each vertex $v \in N_{S}\left(v_{i}\right) \backslash V(T)$ is a pendant vertex of $S$. Since $S$ contains no $3 P_{3}, 2 P_{3} \cup C_{2}, P_{3} \cup 2 C_{2}$, or $3 C_{2}$, there are at most two vertices of $T$ having more than one pendant neighbor or joined to a pendant neighbor by a multiple edge. Therefore, $S$ is contained in some member of $B_{15}$.

2.b There is a triangle $T$ touching a 5-path $P$ at an endpoint of $P$. Let $T=v_{1} v_{2} v_{3} v_{1}$ touch $P=v_{1} w_{1} w_{2} w_{3} w_{4}$ at $v_{1}$. As $S$ contains no $P_{5} \cup P_{3}$ or $P_{5} \cup C_{2}$ and $\ell=3, \hat{d}_{S}\left(v_{2}\right)=\hat{d}_{S}\left(v_{3}\right)=2, N_{S}\left(w_{1}\right) \subseteq\left\{v_{1}, w_{2}, w_{3}\right\}, N_{S}\left(w_{3}\right) \subseteq\left\{w_{1}, w_{2}, w_{4}\right\}, N_{S}\left(w_{4}\right) \subseteq$ $\left\{w_{2}, w_{3}\right\}$, each $v \in N_{S}\left(v_{1}\right) \backslash\left\{v_{2}, v_{3}, w_{1}, w_{2}\right\}$ is a pendant vertex, each $v \in N_{S}\left(w_{2}\right) \backslash\left\{v_{1}, w_{1}, w_{3}, w_{4}\right\}$ is a pendant vertex, and the edges $v_{2} v_{3}$ and $w_{3} w_{4}$ are simple. If $w_{1}$ and $w_{3}$ are nonadjacent, then $S$ is contained in some member of $\mathscr{B}_{16}$. If $w_{1}$ and $w_{3}$ are adjacent, then $w_{2}$ is nonadjacent to $v_{1}$ and to $w_{4}$ because $S$ has no 4 -cycles, and $\hat{d}_{S}\left(w_{2}\right)=2$ because $S$ contains no $P_{5} \cup P_{3}$. Therefore, $S$ is contained in some member of $\mathscr{B}_{10}$.

2.c There are two touching triangles, say $T=v_{1} v_{2} v_{3} v_{1}$ and $T^{\prime}=v_{1} w_{1} w_{2} v_{1}$. By symmetry and since 2.a does not hold, we can assume that $\hat{d}_{S}\left(v_{2}\right)=2$ and $\hat{d}_{S}\left(w_{1}\right)=2$. As $S$ has no 4 -cycles and no bipartite claw, each $v \in N_{S}\left(v_{1}\right) \backslash\left\{v_{2}, v_{3}, w_{1}, w_{2}\right\}$ is a pendant vertex. Since $S$ has no 4-cycles and 2.b does not hold, each $v \in N_{S}\left(v_{3}\right) \backslash V(T)$ is a pendant vertex. Symmetrically, each $v \in N_{S}\left(w_{2}\right) \backslash V\left(T^{\prime}\right)$ is also a pendant vertex.

If each of $v_{1}$ and $w_{2}$ is adjacent to some pendant neighbor, then $v_{2} v_{3}$ is simple and $\hat{d}_{S}\left(v_{3}\right)=2$ (because $S$ contains no $P_{5} \cup C_{2}$ or $P_{5} \cup P_{3}$ ), thus $S$ is contained in some member of $B_{16}$.

So, if $v_{1}$ is adjacent to some pendant neighbor, we can assume that $\hat{d}_{S}\left(v_{3}\right)=\hat{d}_{S}\left(w_{2}\right)=2$ and, since $S$ contains no $P_{3} \cup 2 C_{2}$ or $3 C_{2}$, one of the following conditions hold:

(1) $v_{1}$ is adjacent to exactly one pendant neighbor and the edge joining $v_{1}$ to its pendant neighbor is simple, thus $S$ is contained in $\mathscr{B}_{1}$.

(2) At least one of $v_{2} v_{3}$ and $w_{1} w_{2}$ is simple, which implies that $S$ is contained in a member of $\mathscr{B}_{16}$.

Otherwise, $v_{1}$ is not adjacent to any pendant neighbor. If $w_{2}$ is adjacent to at least two pendant neighbors or there is a multiple edge joining $w_{2}$ to a pendant neighbor, then $v_{2} v_{3}$ is simple and $\hat{d}_{S}\left(v_{3}\right)=2$ (because $S$ contains no 1-braid or 2-braid), thus, as a result, $S$ is contained in some member of $\mathcal{B}_{16}$. Finally, if $w_{2}$ is adjacent to at most one pendant neighbor and any edge joining $w_{2}$ to a pendant vertex is simple and, symmetrically, $v_{3}$ is adjacent to at most one pendant neighbor and any edge joining $v_{3}$ to a pendant vertex is simple, then $S$ is contained in some member of $\mathcal{B}_{2}$.

2.d There is an edge touching two different triangles. Since $S$ has no 4-cycles and 2.c does not hold, any pair of different triangles of $T$ in $S$ are vertex-disjoint. Let $v_{1} w_{1}$ be an edge touching the two triangles $T=v_{1} v_{2} v_{3} v_{1}$ and $T^{\prime}=w_{1} w_{2} w_{3} w_{1}$ in $S$. Since $S$ has no 4-cycle and 2.b does not hold, $\hat{d}_{S}\left(w_{2}\right)=\hat{d}_{S}\left(w_{3}\right)=\hat{d}_{S}\left(v_{2}\right)=\hat{d}_{S}\left(v_{3}\right)=2$. Since $S$ contains no bipartite claw and 2.c does not hold, each $v \in N_{S}\left(v_{1}\right) \backslash\left\{v_{2}, v_{3}, w_{1}\right\}$ is a pendant vertex and also each $v \in N_{S}\left(w_{1}\right) \backslash\left\{w_{2}, w_{3}, v_{1}\right\}$ is a pendant vertex. If none of the edges $v_{2} v_{3}$ and $w_{2} w_{3}$ is multiple, $S$ is contained in some member of $\mathscr{B}_{16}$. If $v_{2} v_{3}$ is multiple, then $w_{2} w_{3}$ is simple (since $S$ contains no 2-braid) and $\hat{d}_{S}\left(v_{1}\right)=3$ (since $S$ contains no $P_{5} \cup C_{2}$ ), and we conclude that $S$ is contained in a member of $\mathscr{B}_{10}$.

2.e There is a triangle $T$ touching $a$ 4-path $P$ at an endpoint of $P$. Let $T=v_{1} v_{2} v_{3} v_{1}$ touch $P=v_{1} w_{1} w_{2} w_{3}$ at $v_{1}$. Since 2.a does not hold, we can assume that $\hat{d}_{S}\left(v_{2}\right)=2$. As 2.c does not hold, $v_{1}$ and $w_{2}$ are nonadjacent. Since $S$ has no 4 -cycles, $v_{1}$ and $w_{3}$ are nonadjacent. As 2.d does not hold, $w_{1}$ and $w_{3}$ are nonadjacent. Since $S$ has no 4 -cycles and no 5-cycles, $v_{3}$ is nonadjacent to $w_{1}, w_{2}$, and $w_{3}$. So, two vertices of $V(T) \cup V(P)$ are adjacent only if they are adjacent in $T$ or in $P$. Since 2.b does not hold, $w_{3}$ is a pendant vertex. As $S$ contains no $P_{3} \cup P_{5}$ and $\ell=3$, there is at most one vertex $v \in N_{S}\left(v_{3}\right) \backslash\left\{v_{1}, v_{2}\right\}$ and, if so, $v$ is a pendant vertex and $v v_{3}$ is simple. Since $S$ has no 4-cycles, 2.c does not hold, and $S$ contains no bipartite claw, each $v \in N_{S}\left(v_{1}\right) \backslash\left\{v_{2}, v_{3}, w_{1}\right\}$ is a pendant vertex. As 2.d does not hold and $S$ contains no bipartite claw, each $v \in N_{S}\left(w_{1}\right) \backslash\left\{v_{1}, w_{2}\right\}$ is a pendant vertex. Since 2.b does not hold, each vertex $v \in N_{S}\left(w_{2}\right) \backslash\left\{w_{1}, w_{3}\right\}$ is a pendant vertex. 
If $w_{2}$ has a pendant neighbor different from $w_{3}$ or $w_{2} w_{3}$ is multiple, then $\hat{d}_{S}\left(w_{1}\right)=\hat{d}_{S}\left(v_{3}\right)=2$ and $v_{2} v_{3}$ is simple (otherwise $S$ contains $P_{5} \cup P_{3}, P_{5} \cup C_{2}$, braid, 1-braid, or 2-braid) and, therefore, $S$ is contained in some member of $\mathscr{B}_{16}$. Hence, we can assume that $\hat{d}_{S}\left(w_{2}\right)=2$ and $w_{2} w_{3}$ is simple.

If $\hat{d}_{S}\left(v_{3}\right)=3$ or $v_{2} v_{3}$ is multiple, then $\hat{d}_{S}\left(v_{1}\right)=3$ (because $S$ contains no $P_{5} \cup P_{3}$ or $\left.P_{5} \cup C_{2}\right)$ and $S$ is contained in some member of $\mathscr{B}_{10}$. Otherwise, $S$ is contained in some member of $\mathcal{B}_{16}$.

2.f None of the previous subcases holds. Let $T=v_{1} v_{2} v_{3} v_{1}$ be a triangle of $S$. Suppose, for the purpose of contradiction, that $v_{1}$ has two non-pendant neighbors different from $v_{2}$ and $v_{3}$. Let $w_{1}, w_{2} \in N_{G}\left(v_{1}\right) \backslash\left\{v_{2}, v_{3}\right\}$ such that $w_{1}$ and $w_{2}$ are non-pendant. Since $w_{1}$ is non-pendant, there exists some vertex $w_{3} \in N_{G}\left(w_{1}\right) \backslash\left\{v_{1}\right\}$. As $S$ has no 4-cycles and 2.c does not hold, $w_{3} \notin V(T) \cup\left\{w_{1}, w_{2}\right\}$. Similarly, there is a vertex $w_{4} \in N_{G}\left(w_{2}\right) \backslash\left\{v_{1}\right\}$ and $w_{4} \notin V(T) \cup\left\{w_{1}, w_{2}, w_{3}\right\}$. But then $S$ contains a bipartite claw, a contradiction.

Hence, each vertex of $T$ is adjacent to at most one non-pendant vertex not in $V(T)$. Since $S$ has no 4-cycles, 2.c and 2.e do not hold, and $\ell=3$, if $w$ is a non-pendant neighbor of $v_{i}$ for some $i \in\{1,2,3\}$, then each $v \in N_{S}(w) \backslash\left\{v_{i}\right\}$ is a pendant vertex.

Suppose that $v_{1}$ has some non-pendant neighbor $w_{1}$ outside $T$ that is adjacent to two pendant neighbors or there is a multiple edge joining $w_{1}$ to a pendant neighbor. Since $S$ contains no $P_{5} \cup P_{3}, P_{5} \cup C_{2}, 3 P_{3}, 2 P_{3} \cup C_{2}, P_{3} \cup 2 C_{2}$, or $3 C_{2}$, if $\hat{d}_{S}\left(v_{1}\right) \geq 4$, then $\hat{d}_{S}\left(v_{2}\right)=\hat{d}_{S}\left(v_{3}\right)=2$ and one of the following holds:

(1) $\hat{d}_{S}\left(v_{1}\right)=4$ and the edge joining $v_{1}$ to a pendant vertex is simple and, consequently, $S$ is contained in some member of $\mathcal{B}_{13}$.

(2) $v_{2} v_{3}$ is simple and $S$ is contained in some member of $\mathscr{B}_{16}$.

So, we assume that $\hat{d}_{S}\left(v_{1}\right)=3$. Since 2 .a does not hold, we can assume that $\hat{d}_{S}\left(v_{3}\right)=2$. Since $S$ contains no $P_{5} \cup P_{3}, P_{5} \cup C_{2}$, braid, 1-braid, or 2-braid, $\hat{d}_{S}\left(v_{2}\right) \leq 3$ and if there is $v \in N_{S}\left(v_{2}\right) \backslash\left\{v_{1}, v_{3}\right\}$, then $v$ is pendant and $v_{2} v$ is simple. We conclude that $S$ is contained in some member of $\mathscr{B}_{10}$.

It only remains to consider the case in which each non-pendant vertex $w \in N_{S}\left(v_{i}\right) \backslash V(T)$ for some $i \in\{1,2,3\}$ satisfies that $\hat{d}_{S}(w)=2$ and that, for each $w^{\prime} \in N_{S}(w) \backslash\left\{v_{i}\right\}, w w^{\prime}$ is simple. Since 2.a does not hold, $S$ is contained in some member of $\mathcal{B}_{16}$.

Case 3. S has a longest cycle of length $\ell=4$. In each subcase, we assume that the previous subcases do not hold.

3.a There are two touching 4-cycles in $S$, say $C=v_{1} v_{2} v_{3} v_{4} v_{1}$ and $C^{\prime}=v_{1} w_{1} w_{2} w_{3} v_{1}$. Since $S$ contains no $P_{3} \cup P_{5}, V(S)=$ $V(C) \cup V\left(C^{\prime}\right)$. Since $S$ contains no $P_{5} \cup C_{2}$, the edges $v_{2} v_{3}, v_{3} v_{4}, w_{1} w_{2}$, and $w_{2} w_{3}$ are simple. If $v_{2} v_{4}$ is multiple, then there is no edge $v_{1} v_{3}$, and if $w_{1} w_{3}$ is multiple, then there is no edge $v_{1} w_{2}$. We conclude that $S$ is contained in some member of $\mathscr{B}_{2}, \mathscr{B}_{3}$, or $\mathscr{B}_{4}$.

3.b There is a triangle $T$ touching a 4-cycle in $S$. Let $C=v_{1} v_{2} v_{3} v_{4} v_{1}$ touch $T=v_{1} w_{1} w_{2} v_{1}$. Since $S$ has no 5-cycle and contains no bipartite claw, necessarily $N_{S}\left(v_{2}\right) \subseteq\left\{v_{1}, v_{3}, v_{4}\right\}, N_{S}\left(v_{4}\right) \subseteq\left\{v_{1}, v_{2}, v_{3}\right\}$, and $N_{S}\left(v_{3}\right) \cap\left\{w_{1}, w_{2}\right\}=\emptyset$. This also means that $N_{S}\left(w_{1}\right) \cap V(C)=N_{S}\left(w_{2}\right) \cap V(C)=\left\{v_{1}\right\}$. Since $S$ contains no $P_{5} \cup P_{3}$ and 3.a does not hold, $\hat{d}_{S}\left(w_{1}\right) \leq 3$ and we can assume that $\hat{d}_{S}\left(w_{2}\right)=2$.

Let us consider the case when $\hat{d}_{S}\left(w_{1}\right)=3$ or $w_{1} w_{2}$ is multiple. Since $S$ contains no $P_{5} \cup P_{3}$ or $P_{5} \cup C_{2}, N_{S}\left(v_{1}\right) \subseteq$ $V(C) \cup V(T), N_{S}\left(v_{3}\right) \subseteq V(C)$, and, if there is some $w_{3} \in N_{S}\left(w_{1}\right) \backslash\left\{v_{1}, w_{2}\right\}$, then $w_{3}$ is a pendant vertex of $S$ and $w_{1} w_{3}$ is simple. In addition, $v_{2} v_{3}$ and $v_{3} v_{4}$ are simple because $S$ contains no 1-braid or 2-braid. If $v_{2} v_{4}$ is not a multiple edge of $S$, then $S$ is contained in some member of $\mathscr{B}_{3}$. Otherwise, $v_{1} v_{3}$ is not an edge of $S$ (because $S$ contains no 1-braid or 2-braid) and $S$ is contained in some member of $\mathscr{B}_{2}$. So, from now on, we assume that $\hat{d}_{S}\left(w_{1}\right)=\hat{d}_{S}\left(w_{2}\right)=2$ and $w_{1} w_{2}$ is simple.

Suppose that $v_{2}$ and $v_{4}$ are adjacent. Since $S$ contains no bipartite claw, each $v \in N_{S}\left(v_{1}\right) \backslash(V(T) \cup V(C))$ is a pendant vertex of $S$. So, if $N_{S}\left(v_{3}\right) \subseteq V(C)$, then $S$ is contained in some member of $\mathcal{B}_{12}$. Therefore, we can assume that there is some $w_{3} \in N_{S}\left(v_{3}\right) \backslash V(C)$. Since $S$ contains no bipartite claw, $P_{5} \cup P_{3}$, or $P_{5} \cup C_{2}, v_{1} v_{3}$ is not an edge of $S,\left|N_{S}\left(v_{3}\right) \backslash V(C)\right|=1, w_{3}$ is a pendant vertex of $S$, and $v_{3} w_{3}$ is simple. We conclude that $S$ is contained in some member of $\mathscr{B}_{11}$.

It only remains to consider the case when $v_{2}$ and $v_{4}$ are nonadjacent. Due to the first remarks of this subcase, $N_{S}\left(v_{2}\right)=N_{S}\left(v_{4}\right)=\left\{v_{1}, v_{3}\right\}$. Notice that each $v \in N_{S}\left(v_{1}\right) \backslash(V(T) \cup V(C))$ satisfies $N_{S}(v) \subseteq\left\{v_{1}, v_{3}\right\}$ because $S$ contains no bipartite claw. If each $v \in N_{S}\left(v_{3}\right) \backslash\left\{v_{1}\right\}$ satisfies that $N_{S}(v) \subseteq\left\{v_{1}, v_{3}\right\}$, then $S$ is contained in some member of $\mathcal{B}_{16}$. So, we can assume that there is some $w_{3} \in N_{S}\left(v_{3}\right) \backslash\left\{v_{1}\right\}$ and some $w_{4} \in N_{S}\left(w_{3}\right) \backslash\left\{v_{1}, v_{3}\right\}$. By construction, $w_{3}, w_{4} \notin V(C) \cup V(T)$. Then, $N_{S}\left(w_{3}\right)=\left\{v_{3}, w_{4}\right\}$ and $w_{3} w_{4}$ is simple since $S$ contains no bipartite claw, braid or 1-braid. In addition, $N_{S}\left(w_{4}\right) \subseteq\left\{v_{3}, w_{3}\right\}$ because $S$ contains no $P_{3} \cup P_{5}$. Since $S$ contains no bipartite claw, each $v \in N_{S}\left(v_{3}\right) \backslash\left\{v_{1}, v_{2}, v_{4}, w_{3}, w_{4}\right\}$ satisfies $N_{S}(v) \subseteq\left\{v_{1}, v_{3}\right\}$. Thus, $S$ is contained in some member of $\mathcal{B}_{16}$.

3.c $S$ contains $K_{2,3}$. Equivalently, suppose that there are two vertices $v_{1}, v_{3} \in V(S)$ such that $N_{S}\left(v_{1}\right) \cap N_{S}\left(v_{3}\right)$ consists of at least three vertices. Let $v_{2}$ be a vertex of $N_{S}\left(v_{1}\right) \cap N_{S}\left(v_{3}\right)$ of maximum degree in $\widehat{S}$ and let $v_{4}$ and $v_{5}$ be any two other vertices of $N_{S}\left(v_{1}\right) \cap N_{S}\left(v_{3}\right)$. Since $\ell=4$ and $S$ contains no bipartite claw, $\left\{v_{2}, v_{4}, v_{5}\right\}$ is a stable set, $\hat{d}_{S}\left(v_{4}\right)=\hat{d}_{S}\left(v_{5}\right)=2$, and each $v \in N_{S}\left(v_{2}\right) \backslash\left\{v_{1}, v_{3}\right\}$ is a pendant vertex.

Suppose that each vertex $v \in\left(N_{S}\left(v_{1}\right) \cup N_{S}\left(v_{3}\right)\right) \backslash\left\{v_{1}, v_{2}, v_{3}\right\}$ is such that $N_{S}(v) \subseteq\left\{v_{1}, v_{3}\right\}$. If $v_{2}$ is adjacent to at most one pendant vertex and any edge joining $v_{2}$ to a pendant vertex is simple, then $S$ is contained in some member of $\mathcal{B}_{15}$. So, assume, on the contrary, that $v_{2}$ is adjacent to at least two pendant vertices or $v_{2}$ is joined to a pendant vertex through 
a multiple edge. Then, $N_{S}\left(v_{1}\right) \subseteq\left\{v_{2}, v_{3}, v_{4}, v_{5}\right\}$ and $N_{S}\left(v_{3}\right) \subseteq\left\{v_{1}, v_{2}, v_{4}, v_{5}\right\}$ (because $S$ contains no $P_{5} \cup P_{3}$ or $P_{5} \cup C_{2}$ ), each of the edges $v_{1} v_{4}, v_{1} v_{5}, v_{3} v_{4}, v_{3} v_{5}$ is simple (because $S$ contains no 1-braid and no 2-braid) and, consequently, $S$ is contained in some member of $\mathscr{B}_{14}$. So, we can suppose that there is some vertex $w_{1} \in N_{S}\left(v_{1}\right) \backslash\left\{v_{2}, v_{3}\right\}$ such that $N_{S}\left(w_{1}\right) \nsubseteq\left\{v_{1}, v_{3}\right\}$ and let $w_{2} \in N_{S}\left(w_{1}\right) \backslash\left\{v_{1}, v_{3}\right\}$. Since $S$ contains no $P_{3} \cup P_{5}, \hat{d}_{S}\left(v_{2}\right)=2$. Notice that, by construction, $w_{1}$ is nonadjacent to $v_{3}$; otherwise, $w_{1} \in N_{S}\left(v_{1}\right) \cap N_{S}\left(v_{3}\right)$ and $\hat{d}_{S}\left(w_{1}\right)>2=\hat{d}_{S}\left(v_{2}\right)$, contradicting the choice of $v_{2}$. Since $S$ contains no braid or 1-braid, $\hat{d}_{S}\left(w_{1}\right)=2$ and $w_{1} w_{2}$ is simple. Notice that $w_{2}$ is a pendant vertex because $S$ has no 5 -cycle, contains no $P_{5} \cup P_{3}$, and 3.b does not hold. Since $S$ contains no bipartite claw, $w_{1}$ is the only vertex $v \in N_{S}\left(v_{1}\right) \backslash\left\{v_{2}, v_{3}\right\}$ such that $N_{S}(v)$ is not contained in $\left\{v_{1}, v_{3}\right\}$. By symmetry, there is at most one vertex $w_{3} \in N_{S}\left(v_{3}\right) \backslash\left\{v_{1}, v_{2}\right\}$ such that $N_{S}\left(w_{3}\right) \nsubseteq\left\{v_{1}, v_{3}\right\}$ and, if so, $\hat{d}_{S}\left(w_{3}\right)=2$, the only vertex $w_{4} \in N_{S}\left(w_{3}\right) \backslash\left\{v_{3}\right\}$ is pendant, and $w_{3} w_{4}$ is simple. Since, by construction, all vertices $v \in\left(N_{S}\left(v_{1}\right) \cup N_{S}\left(v_{3}\right)\right) \backslash\left\{v_{1}, v_{3}, w_{1}, w_{3}\right\}$ are such that $N_{S}(v) \subseteq\left\{v_{1}, v_{3}\right\}, S$ is contained in some member of $\mathscr{B}_{16}$.

3.d There is a 4-cycle $C=v_{1} v_{2} v_{3} v_{4} v_{1}$ such that each vertex $v_{i}$ of $C$ has a neighbor $w_{i} \notin V(C)$. Since $S$ has no 5-cycle and 3.c does not hold, $N_{S}\left(v_{i}\right) \cap N_{S}\left(v_{j}\right) \subseteq V(C)$ for all $i$ and all $j$. In particular, $w_{1}, w_{2}, w_{3}$, and $w_{4}$ are pairwise different. Since $S$ contains no $P_{5} \cup P_{3}$ or $P_{5} \cup C_{2}, w_{i}$ is the only vertex in $N_{S}\left(v_{i}\right) \backslash V(C)$ and $v_{i} w_{i}$ is simple for each $i=1,2$, 3, 4. Moreover, $w_{1}, w_{2}, w_{3}$, and $w_{4}$ are pendant vertices as $S$ has no 6 -cycle and no bipartite claw. Finally, since $S$ contains no bipartite claw, $C$ is chordless and we conclude that $S$ is a member of $\mathscr{B}_{5}$.

3.e There is a 4-cycle $C$ touching a 4-path $P$ at an endpoint of $P$. Let $C=v_{1} v_{2} v_{3} v_{4} v_{1}$ touch $P=v_{1} w_{1} w_{2} w_{3}$ in $v_{1}$. Since $\ell=4, S$ contains no $P_{5} \cup P_{3}$ or $P_{5} \cup C_{2}$, and 3.a does not hold, $N_{S}\left(w_{3}\right) \subseteq\left\{w_{1}, w_{2}\right\}$ and $w_{2} w_{3}$ is simple. Similarly, and since 3.b does not hold, $N_{S}\left(w_{2}\right)=\left\{w_{1}, w_{3}\right\}$. As $S$ has no 5-cycles and 3.c does not hold, $N_{S}\left(w_{1}\right) \cap V(C)=\left\{v_{1}\right\}$. Since $\ell=4$ and $S$ contains no $P_{5} \cup P_{3}$, each $v \in N_{S}\left(w_{1}\right) \backslash\left\{v_{1}, w_{2}, w_{3}\right\}$ is a pendant vertex of $S, N_{S}\left(v_{1}\right) \subseteq V(C) \cup\left\{w_{1}\right\}$, and $N_{S}\left(v_{2}\right), N_{S}\left(v_{3}\right), N_{S}\left(v_{4}\right) \subseteq V(C)$. Notice also that $v_{2} v_{3}$ and $v_{3} v_{4}$ are simple because $S$ contains no $P_{5} \cup C_{2}$. Therefore, if $v_{2} v_{4}$ is not a multiple edge of $S$, then $S$ is contained in some member of $\mathcal{B}_{9}$. If, on the contrary, $v_{2} v_{4}$ is multiple, then $v_{1}$ and $v_{3}$ are nonadjacent (because $S$ contains no $P_{5} \cup C_{2}$ ) and $S$ is contained in some member of $\mathscr{B}_{10}$.

3.f There is a 4-cycle $C=v_{1} v_{2} v_{3} v_{4} v_{1}$ such that three of its vertices have a neighbor outside $C$, say, $v_{i}$ has a neighbor $w_{i} \notin V(C)$ for each $i=1$, 2, 3. Then, $N_{S}\left(v_{1}\right) \backslash V(C), N_{S}\left(v_{2}\right) \backslash V(C)$, and $N_{S}\left(v_{3}\right) \backslash V(C)$ are pairwise disjoint and each $w \in N_{S}\left(v_{i}\right) \backslash V(C)$, for each $i \in\{1,2,3\}$, is a pendant vertex because 3.c does not hold and $S$ has no 5 -cycles or 6 -cycle and contains no $P_{5} \cup P_{3}$. Since 3.d does not hold and $S$ contains no bipartite claw, $N_{S}\left(v_{4}\right)=\left\{v_{1}, v_{3}\right\}$. Finally, $w_{2}$ is the only pendant neighbor of $v_{2}$ and $v_{2} w_{2}$ is simple because $S$ contains no $P_{5} \cup P_{3}$ or $P_{5} \cup C_{2}$. We conclude that $S$ is contained in some member of $\mathscr{B}_{15}$.

3.g There is a 4-cycle $C=v_{1} v_{2} v_{3} v_{4} v_{1}$ where $v_{1}$ is adjacent to a non-pendant vertex $w_{1} \notin V(C)$. Let $w_{2}$ be any vertex of $N_{S}\left(w_{1}\right) \backslash\left\{v_{1}\right\}$. Then, $w_{2} \notin V(C)$ because $S$ contains no 5-cycle and 3.c does not hold. As $S$ has no 5-cycle or 6-cycle and 3.b does not hold, $N_{S}\left(w_{2}\right) \cap V(C)=\emptyset$. Therefore, $w_{2}$ is a pendant vertex as 3.e does not hold. Notice that $N_{S}\left(v_{2}\right), N_{S}\left(v_{4}\right) \subseteq V(C)$ because $S$ contains no bipartite claw. Since $w_{2}$ is an arbitrary vertex of $N_{S}\left(w_{1}\right) \backslash\left\{v_{1}\right\}$, each $w \in N_{S}\left(w_{1}\right) \backslash\left\{v_{1}\right\}$ is a pendant vertex. Since $w_{1}$ is an arbitrary non-pendant vertex in $N_{S}\left(v_{1}\right) \backslash V(C)$, for every nonpendant vertex $w_{1}^{\prime}$ in $N_{S}\left(v_{1}\right) \backslash V(C)$, each $w \in N_{S}\left(w_{1}^{\prime}\right) \backslash\left\{v_{1}\right\}$ is a pendant vertex. Thus, since $S$ contains no $P_{3} \cup P_{5}, w_{1}$ is the only non-pendant vertex in $N_{S}\left(v_{1}\right) \backslash V(C)$; i.e., each $v \in N_{S}\left(v_{1}\right) \backslash\left\{v_{2}, v_{3}, v_{4}, w_{1}\right\}$ is a pendant vertex.

Suppose first that $\hat{d}_{S}\left(w_{1}\right)>2$ or $w_{1} w_{2}$ is multiple. Since $S$ contains no $P_{5} \cup P_{3}$ or $P_{5} \cup C_{2}, v_{1}$ has no pendant neighbors and $N_{S}\left(v_{3}\right) \subseteq V(C)$. Moreover, $v_{2} v_{3}$ and $v_{3} v_{4}$ are simple because $S$ contains no 1-braid or 2-braid. If $v_{2} v_{4}$ is not a multiple edge, then $S$ is contained in some member of $\mathcal{B}_{9}$, but if $v_{2} v_{4}$ is a multiple edge, then $v_{1} v_{3}$ is not an edge of $S$ (because $S$ contains no 1-braid or 2-braid) and $S$ is contained in some member of $\mathcal{B}_{10}$. So, from now on, let $\hat{d}_{S}\left(w_{1}\right)=2$ and $w_{1} w_{2}$ be simple.

Suppose that $v_{2}$ and $v_{4}$ are adjacent. If $v_{3}$ is adjacent to some $v \in V(S) \backslash V(C)$, then $v$ is the only such vertex, $v$ is pendant, and $v_{3} v$ is simple (because $S$ contains no $P_{5} \cup P_{3}$ or $P_{5} \cup C_{2}$ ) and $v_{1}$ is not adjacent to $v_{3}$ (because $S$ contains no bipartite claw), so $S$ is contained in some member of $\mathscr{B}_{11}$. Otherwise, $S$ is contained in some member of $\mathscr{B}_{12}$. So, from now, we assume that $v_{2}$ and $v_{4}$ are nonadjacent.

If $v_{3}$ also has some non-pendant neighbor $w_{3} \in V(S) \backslash V(C)$, the same argumentation as for $w_{1}$ implies that each $v \in N_{S}\left(v_{3}\right) \backslash V(C)$ different from $w_{3}$ is pendant and we can assume that $\hat{d}_{S}\left(w_{3}\right)=2$ and, if $w_{4}$ is the only vertex of $N_{S}\left(w_{3}\right) \backslash\left\{v_{3}\right\}$, then $w_{3} w_{4}$ is simple. Thus, $S$ is contained in some member of $\mathscr{B}_{16}$, even if $v_{3}$ has no non-pendant neighbor.

3.h None of the previous subcases holds. Since $\ell=4$, there exists some 4-cycle $C=v_{1} v_{2} v_{3} v_{4} v_{1}$ in $S$. Since 3.g does not hold, each $v \in N_{S}\left(v_{i}\right) \backslash V(C)$ is pendant, for each $i=1,2,3$, 4. Since 3.f does not hold, there are at most two vertices of $C$ that are adjacent to pendant vertices. If there are less than two vertices of $V(C)$ adjacent to pendant vertices, $S$ is contained in some member of $\mathcal{B}_{13}$. Therefore, we assume that there are two vertices of $V(C)$ adjacent to pendant vertices, say $v_{1}$ and $v_{j}$ where $j=2$ or $j=3$.

If each of the vertices $v_{1}$ and $v_{j}$ is adjacent to two pendant vertices or joined to some pendant vertex through a multiple edge, then $j=3$ and $v_{2}$ is nonadjacent to $v_{4}$ (since $S$ contains no braid, 1-braid, or 2-braid). We conclude that $S$ is contained in some member of $\mathcal{B}_{16}$.

Finally, if $v_{j}$ is adjacent to only one pendant vertex through a simple edge, then $S$ is contained in some member of $B_{13}$.

Case 4. $S$ has a longest cycle $C$ of length $\ell=6$, say $C=v_{1} v_{2} v_{3} v_{4} v_{5} v_{6} v_{1}$. Since $S$ is connected and contains no 6-pan, the vertices of $C$ are the only vertices of $S$. As $S$ contains no 5 -cycle, $C$ has no short chords. 
Suppose first that $C$ has two multiple chords, say $v_{1} v_{4}$ and $v_{2} v_{5}$ are multiple edges. Since $S$ contains no 2-braid, there is no edge $v_{3} v_{6}$ in $S$ and each of $v_{2} v_{3}, v_{3} v_{4}, v_{5} v_{6}$, and $v_{6} v_{1}$ is simple. This means that $S$ is a member of $\mathcal{B}_{7}$. So, from now on, we can assume that $C$ has at most one multiple chord.

Since $C$ has at most one multiple edge, $S$ would belong to $\mathscr{B}_{8}$ if no edge of $C$ was multiple. Therefore, assume from now on that $v_{1} v_{2}$ is multiple. As $S$ contains no 2-braid, none of $v_{3} v_{4}$ and $v_{5} v_{6}$ is multiple and at most one of $v_{1} v_{6}, v_{2} v_{3}, v_{5} v_{6}$ is multiple. In turn, if $C$ has no multiple chords, then $S$ is a member of $\mathscr{B}_{7}$ or $\mathcal{B}_{8}$. So, from now on, let $C$ have exactly one multiple chord.

Since $S$ contains no 2-braid, $S$ is a member of $\mathcal{B}_{6}$ if $v_{3} v_{6}$ is the only multiple chord of $C$ because $v_{4} v_{5}$ cannot be multiple, $v_{1}$ cannot be adjacent to $v_{4}$, and $v_{2}$ cannot be adjacent to $v_{5}$. By symmetry, assume that the only chord of $C$ is $v_{1} v_{4}$. Recall that the only possible multiple edges of $C$ are $v_{1} v_{6}, v_{2} v_{3}$, and $v_{4} v_{5}$ and that at most one of them is multiple. If $v_{1} v_{6}$ is multiple, then $S$ is a member of $\mathscr{B}_{8}$. If $v_{4} v_{5}$ is multiple, then there is no edge $v_{3} v_{6}$ in $S$ (because $S$ contains no 2-braid) and, consequently, $S$ is a member of $\mathscr{B}_{7}$. If $v_{2} v_{3}$ is multiple, then $v_{2} v_{5}$ and $v_{3} v_{6}$ are not edges of $S$ (because $S$ contains no 2-braid) and, consequently, $S$ is a member of $\mathcal{B}_{6}$. Finally, if none of $v_{4} v_{5}, v_{1} v_{6}$, and $v_{2} v_{3}$ is multiple, then $S$ is a member of $\mathcal{B}_{8}$.

Hence, any component $S$ of $H$ is contained in some member of $\mathscr{B}_{1}, \ldots, \mathscr{B}_{16}$. Consequently, (v).(b) holds, which completes the proof.

\subsection{Recognizing balanced complements of line graphs}

We will derive, from the above theorem, the existence of a linear-time recognition algorithm for balanced graphs within complements of line graphs of multigraphs.

Given a graph $G$, we define a pruned graph of $G$ as any maximal induced subgraph of $G$ having no three pairwise false twins and no universal vertices. Let $V_{1}, V_{2}, \ldots, V_{q}$ be the equivalence classes of the relation "is a false twin of" on the set of vertices of $G$. We say that the equivalence class $V_{i}$ is universal if some vertex of $V_{i}$ is a universal vertex of $G$. Clearly, if $V_{i}$ is universal, then $\left|V_{i}\right|=1$. The pruned graphs of $G$ are those subgraphs of $G$ induced by some set $V_{1}^{\prime} \cup V_{2}^{\prime} \cup \ldots \cup V_{q}^{\prime}$ such that $V_{i}^{\prime} \subseteq V_{i}$ and $\left|V_{i}^{\prime}\right|=\beta_{i}$, for each $i=1,2, \ldots, q$, where

$$
\beta_{i}= \begin{cases}\min \left(\left|V_{i}\right|, 2\right) & \text { if } V_{i} \text { is not universal } \\ 0 & \text { otherwise. }\end{cases}
$$

Since any two vertices that belong to the same $V_{i}$ are nonadjacent and have the same neighbors, the pruned graphs of $G$ are unique up to isomorphisms and we denote any of them by $\mathcal{P}(G)$.

Lemma 19. A pruned subgraph of a graph $G$ can be computed in linear time.

Proof. In order to compute $\mathcal{P}(G)$, we first construct the modular decomposition tree of $G$. We omit the details of this data structure but refer the reader to $[9,12,23]$, where linear-time algorithms to build such a decomposition tree are given. Two vertices $u$ and $v$ of $G$ are false twins if and only if the leaves of the modular decomposition tree representing them are children of the same parallel node. This means that we can find a subset of vertices inducing a pruned graph of $G$ by marking for exclusion all universal vertices of $G$ and performing a breadth-first search on the modular decomposition tree of $G$ and also marking for exclusion the third, fourth, fifth, and so on, leaf children of each parallel node. Since the modular decomposition tree can be computed in linear time, $\mathcal{P}(G)$ can also be computed in linear time.

The following fact about $\mathcal{P}(G)$ is crucial for our purposes.

Corollary 20. Let $G$ be the complement of the line graph of a multigraph. Then, $G$ is balanced if and only if $\mathcal{P}(G)$ is balanced.

Proof. If $G$ is balanced, then clearly $\mathcal{P}(G)$ is also balanced (because $\mathcal{P}(G)$ is an induced subgraph of $G$ ). In order to prove the converse, we assume that $G$ is not balanced and we will prove that $\mathscr{P}(G)$ is not balanced. Let $W$ be a subset of vertices inducing a minimal induced subgraph of $G$ that is not balanced. By Theorem 18, the subgraph of $G$ induced by $W$ is isomorphic to 3-sun, 2-pyramid, 3-pyramid, $C_{5}, C_{7}, U_{7}$, or $V_{7}$. In particular, there are no three pairwise false twins of $G$ in $W$ and there is no universal vertex of $G$ in $W$. Therefore, if the equivalence classes $V_{1}, V_{2}, \ldots, V_{q}$ and $\beta_{i}$ are as defined earlier and $W_{i}=W \cap V_{i}$, then $\left|W_{i}\right| \leq \beta_{i}$ for each $i=1,2, \ldots, q$. So, it is possible to find $V_{1}^{\prime}, V_{2}^{\prime}, \ldots, V_{q}^{\prime}$ such that $W_{i} \subseteq V_{i}^{\prime} \subseteq V_{i}$ and $\left|V_{i}^{\prime}\right|=\beta_{i}$ for each $i=1,2, \ldots, q$. Then, $G^{\prime}=G\left[V_{1}^{\prime} \cup V_{2}^{\prime} \cup \cdots \cup V_{q}^{\prime}\right]$ is a pruned graph of $G$ and $G^{\prime}$ is not balanced because $W \subseteq V\left(G^{\prime}\right)$ and $G^{\prime}[W]=G[W]$ is not balanced.

Let $G$ be the complement of the line graph of a multigraph and let $k$ be a fixed integer. According to Corollary 20, if $\mathcal{P}(G)$ has at most $k$ vertices, we can decide whether $G$ is balanced in linear time by computing $\mathcal{P}(G)$ in linear time and then deciding whether $\mathcal{P}(G)$ is balanced in constant time. (Indeed, the obvious $O\left(n^{7}\right)$-time algorithm that follows from assertion (iii) of Theorem 18 becomes constant-time when $n=O(1)$.) In what follows, we will fix $k=40$ and the remainder of this subsection is devoted to proving that we can decide in linear time whether $\mathcal{P}(G)$ is balanced even if $\mathcal{P}(G)$ has more than 40 vertices.

If $G$ is the line graph of a multigraph, we denote by $L^{-1}(G)$ any multigraph $H$ without isolated vertices such that $L(H)=G$ and whose underlying graph $\widehat{H}$ satisfies $L(\widehat{H})=\mathcal{R}(G)$, where $\mathcal{R}(G)$ is the representative graph of $G$ as defined near the end 


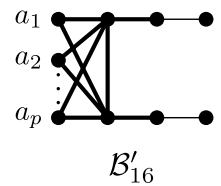

Fig. 8. Multigraph family $\mathfrak{B}_{16}^{\prime}$. Light lines represent single edges, whereas bold lines represent one or more parallel edges. Parameter $p$ varies over the positive integers, and $a_{1}, a_{2}, \ldots, a_{p}$ are pairwise false twins.

of Section 3. Given a graph $G$, a multigraph $L^{-1}(G)$ can be computed in linear time of $G$ (see [15, pp. 67-68]). We say that two incident edges $e_{1}$ and $e_{2}$ of a multigraph $H$ are twins if they are incident to the same edges of $E(H)$. We say that a multigraph $H$ is reduced if each pair of twin edges are parallel. By definition, $H=L^{-1}(G)$ is reduced. In Fig. 8 we introduce the multigraph family $\mathcal{B}_{16}^{\prime}$.

Corollary 21. Let $G$ be the complement of the line graph of a multigraph and suppose that $\mathcal{P}(G)$ has more than 40 vertices. If $H=L^{-1}(\overline{P(G)})$, then the following conditions are equivalent:

(i) $G$ is balanced.

(ii) $H$ is a connected submultigraph of some member of $\mathscr{B}_{15}$ or $\mathcal{B}_{16}^{\prime}$.

(iii) $H$ is connected, has exactly two vertices $v_{1}$ and $v_{2}$ that are incident to at least six edges each, and, for each $i=1,2$, there is at most one neighbor $w_{i}$ of $v_{i}$ such that there is some $x_{i} \in N_{H}\left(w_{i}\right) \backslash\left\{v_{1}, v_{2}\right\}$ and, if so, each of the following holds: $N_{H}\left(w_{i}\right) \subseteq\left\{x_{i}, v_{1}, v_{2}\right\}$, there is exactly one edge $e_{i}$ joining $w_{i}$ to $x_{i}$, and $e_{i}$ is the only edge incident to $x_{i}$. Moreover, if $w_{1}$ and $w_{2}$ exist and are different, then $N_{G}\left(w_{i}\right)=\left\{v_{i}, x_{i}\right\}$ for each $i=1,2$.

Proof. Suppose that $G$ is balanced and let $H=L^{-1}(\overline{\mathcal{P}(G)})$. As $H$ has no isolated vertices and $\mathcal{P}(G)$ has no universal vertices, each component of $H$ has at least two edges. Since $G$ is balanced, $\mathcal{P}(G)$ is balanced; i.e., $H$ is $\bar{L}$-balanced. So, by Theorem 18 , either $H$ is a connected submultigraph of some member of $\mathcal{B}_{1}, \mathcal{B}_{2}, \ldots, \mathcal{B}_{16}$ or $H$ has two components, each of which is contained in a member of $\mathcal{A}_{1}, \mathcal{A}_{2}$, or $\mathcal{A}_{3}$. But, as $\mathcal{P}(G)$ has more than 40 vertices, $H$ has more than 40 edges. Since, by construction, $\bar{P}(G)$ has no three pairwise true twins, $H$ has no three pairwise parallel edges. Since, in addition, $H$ is reduced, $H$ is necessarily a connected submultigraph of $\mathscr{B}_{15}$ or $\mathscr{B}_{16}^{\prime}$. Conversely, if $H$ is a submultigraph of some member of $\mathscr{B}_{15}$ or $\mathcal{B}_{16}^{\prime}$, then $\mathcal{P}(G)$ is balanced by Theorem 18 and, then, $G$ is also balanced by Corollary 20 . This concludes the proof of the equivalence between (i) and (ii).

Since clearly (iii) implies (ii), it only remains to show that (ii) implies (iii). So, assume that $H$ is a connected submultigraph of some member of $\mathcal{B}_{15}$ or $\mathscr{B}_{16}^{\prime}$. Since $H=L^{-1}(\overline{\mathcal{P}(G)}), H$ has no three pairwise parallel edges. Therefore, $H$ has at most two vertices incident to at least six edges. Moreover, since $H$ has at least 40 edges, $H$ has exactly two vertices incident to at least six edges each, and (iii) clearly holds.

The next result implies that if $\mathcal{P}(G)$ has more than 40 vertices, then we can either detect that $G$ is not balanced or compute $L^{-1}(\overline{\mathcal{P}(G)})$ efficiently.

Corollary 22. Let $G$ be the complement of the line graph of a multigraph. Let $n_{\mathcal{P}}$ and $m_{\mathcal{P}}$ be the number of vertices and edges of $\mathcal{P}(G)$ and suppose that $n_{\mathcal{P}}>40$. If

$$
m_{\mathcal{P}} \geq \frac{2}{9}\left(n_{\mathcal{P}}-3\right)\left(n_{\mathcal{P}}-36\right)
$$

does not hold, then $G$ is not balanced. On the other hand, if $(*)$ holds, then $H=L^{-1}(\overline{\mathcal{P}(G)})$ can be computed from $G$ in linear time.

Proof. Suppose first that $G$ is balanced and let $H=L^{-1}(\overline{\mathcal{P}(G)})$. Then, $H$ has $n_{\mathcal{P}}$ edges and satisfies condition (iii) of Corollary 21. Let $A$ be the set of vertices $a$ of $H$ such that $N_{H}(a) \subseteq\left\{v_{1}, v_{2}\right\}$. Since $\overline{\mathcal{P}(G)}$ has no three pairwise true twins, $H$ has no three pairwise parallel edges. Moreover, as $H$ is reduced, there are at most two edges joining $v_{i}$ to pendant vertices in $A$, for each $i=1,2$. Let $E_{i}$ be the set of edges joining $v_{i}$ to non-pendant vertices in $A$, for each $i=1,2$. Since $H$ is a submultigraph of a member of $\mathcal{B}_{15}$ or $\mathscr{B}_{16}^{\prime}$ and $H$ is reduced, $\left|E_{1}\right|+\left|E_{2}\right| \geq n_{\mathcal{P}}-12$. Without loss of generality, assume that $\left|E_{1}\right| \geq\left|E_{2}\right|$. Then, $\frac{1}{2}\left(n_{\mathcal{P}}-12\right) \leq\left|E_{1}\right| \leq \frac{2}{3} n_{\mathcal{P}}$ because each non-pendant vertex of $A$ is joined to $v_{1}$ by at most two edges and joined to $v_{2}$ by at least one edge. So, since each edge of $E_{2}$ is incident to at most two edges of $E_{1}$ and $\mathcal{P}(G)=\overline{L(H)}$,

$$
m_{\mathcal{P}} \geq\left|E_{2}\right|\left(\left|E_{1}\right|-2\right) \geq\left(n_{\mathcal{P}}-12-\left|E_{1}\right|\right)\left(\left|E_{1}\right|-2\right) \geq \frac{2}{9}\left(n_{\mathcal{P}}-3\right)\left(n_{\mathcal{P}}-36\right) .
$$

This proves that if $(*)$ does not hold, then $G$ is not balanced.

Suppose now that $(*)$ holds. We have seen that $\mathcal{P}(G)$ can be computed in $O(m+n)$ time, where $n$ and $m$ are the number of vertices and edges of $G$. The complement of $\mathcal{P}(G)$ can obviously be computed in $O\left(n_{\mathcal{P}}^{2}\right)$ time. In addition, $H=L^{-1}(\overline{\mathcal{P}(G)})$ can be computed from $\overline{\mathcal{P}(G)}$ in linear time of $\overline{\mathcal{P}(G)}$, which is again $O\left(n_{\mathcal{P}}^{2}\right)$. Notice that since $m_{\mathcal{P}} \leq m$ and we are assuming that $(*)$ holds, $O\left(n_{\mathcal{P}}^{2}\right)$ is $O(m)$. We conclude that $H$ can be computed from $G$ in $O(m+n)$ time, as desired. 
Let $G$ be the complement of the line graph of a multigraph. We know that if $\mathcal{P}(G)$ has at most 40 vertices, we can decide whether $G$ is balanced in linear time. So, suppose that $\mathcal{P}(G)$ has more than 40 vertices and let $n_{\mathcal{P}}$ and $m_{\mathcal{P}}$ be the number of vertices and edges of $\mathcal{P}(G)$. If (*) does not hold, we know that $G$ is not balanced. Otherwise, we can decide whether $G$ is balanced in linear time by first computing $H=L^{-1}(\overline{\mathcal{P}(G)})$ and then checking the validity of condition (iii) of Corollary 21 . We conclude:

Corollary 23. Given a graph $G$ that is the complement of the line graph of a multigraph, it can be decided whether $G$ is balanced in linear time.

\section{Acknowledgments}

The authors would like to thank the anonymous reviewers for their valuable corrections and suggestions that improved the presentation of this paper.

The first author was partially supported by ANPCyT PICT 2007-00518 and 2007-00533, and UBACyT Grants 20020100100980 and 20020090300094 (Argentina). The second author was partially supported by FONDECyT Grant 1110797 and the Millennium Science Institute "Complex Engineering Systems” (Chile), and ANPCyT PICT 2007-00518, UBACyT Grant 20020100100980, and CONICET PIP 112-200901-00178 (Argentina). The third author was partially supported by ANPCyT PICT 2007-00518, UBACyT Grant 20020100100980, and CONICET PIP 112-200901-00178 (Argentina).

\section{References}

[1] C. Berge, Färbung von Graphen, deren sämtliche beziehungsweise, deren ungerade Kreise starr sind (Zusammenfassung), Wiss. Z. Martin-Luther-Univ. Halle-Wittenberg, Math.-Naturwiss. Reihe 10 (1961) 114-115.

[2] C. Berge, Balanced matrices, Math. Program. 2 (1972) 19-31.

[3] C. Berge, Minimax relations for the partial q-colorings of a graph, Discrete Math. 74 (1989) 3-14

[4] C. Berge, V. Chvátal, Introduction, in: Topics on Perfect Graphs, in: North-Holland Mathematics Studies, vol. 88, Noth-Holland, 1984, pp. vii-xiv.

[5] F. Bonomo, G. Durán, M.C. Lin, J.L. Szwarcfiter, On balanced graphs, Math. Program. 105 (2006) 233-250.

[6] M. Chudnovsky, G.P. Cornuéjols, X. Liu, P.D. Seymour, K. Vušković, Recognizing Berge graphs, Combinatorica 25 (2005) 143-186.

[7] M. Chudnovsky, N. Robertson, P.D. Seymour, R. Thomas, The strong perfect graph theorem, Ann. of Math. 164 (2006) 51-229.

[8] V. Chvátal, On certain polytopes associated with graphs, J. Combin. Theory Ser. B 18 (1975) 138-154.

[9] A. Cournier, M. Habib, A new linear algorithm for modular decomposition, in: S. Tison (Ed.), Proc. 19th International Colloquium on Trees in Algebra and Programming, CAAP'94, Edinburgh, U.K., April 1994, in: Lect. Notes Comput. Sci., vol. 787, Springer, 1994, pp. 68-84.

[10] D.R. Fulkerson, A.J. Hoffman, R. Oppenheim, On balanced matrices, in: M. Balinski (Ed.), Pivoting and Extensions: In Honor of A.W. Tucker, in: Mathematical Programming Study, vol. 1, North-Holland, Amsterdam, 1974, pp. 120-133.

[11] M.C. Golumbic, Trivially perfect graphs, Discrete Math. 24 (1978) 105-107.

[12] M. Habib, F. de Montgolfier, C. Paul, A simple linear-time modular decomposition algorithm, Technical Report RR-LIRMM-03007, LIRMM, Université de Montpellier 2, Montepellier, France, 2003.

[13] P.L. Hammer, Difference graphs, Discrete Appl. Math. 28 (1990) 35-44.

[14] P. Heggernes, D. Kratsch, Linear-time certifying recognition algorithms and forbidden induced subgraphs, Nord. J. Comput. 14 (2007) 87-108.

[15] A. King, Claw-free graphs and two conjectures on omega, delta, and chi, Ph.D. Thesis, School of Computer Science, McGill University, Montreal, Canada, 2009.

[16] T. Kloks, D. Kratsch, H. Müller, Dominoes, in: E.W. Mayr, G. Schmidt, G. Tinhofer (Eds.), Proc. 20th International Workshop on Graph-Theoretic Concepts in Computer Science, WG'94, Herrshing, Germany, June 1994, in: Lect. Notes Comput. Sci., vol. 903, Springer, 1995, pp. 106-120.

[17] D. König, Über Graphen und ihre Anwendung auf Determinantentheorie und Mengenlehre, Math. Ann. 77 (1916) 453-465.

[18] D. Kőnig, Graphok és Matrixok, Mat. Fiz. Lapok 38 (1931) 116-119.

[19] P.G.H. Lehot, An optimal algorithm to detect a line graph and output its root graph, J. ACM 21 (1974) 569-575.

[20] M.C. Lin, J.L. Szwarcfiter, Faster recognition of clique-Helly and hereditary clique-Helly graphs, Inform. Process. Lett. 3 (2007) 40-43.

[21] L. Lovász, Normal hypergraphs and the perfect graph conjecture, Discrete Math. 2 (1972) 253-267.

[22] V.V. Lozin, E-free bipartite graphs, Diskretn. Anal. Issled. Oper. Ser. 17 (2000) 49-66 (in Russian).

[23] R.M. McConnell, J.P. Spinrad, Modular decomposition and transitive orientation, Discrete Math. 201 (1999) 189-241.

[24] E. Prisner, Hereditary clique-Helly graphs, J. Combin. Math. Combin. Comput. 14 (1993) 216-220.

[25] N.D. Roussopoulos, A $\max \{m, n\}$ algorithm for determining the graph $H$ from its line graph $G$, Inform. Process. Lett. 2 (1973) $108-112$.

[26] R.E. Tarjan, Depth-first search and linear graph algorithms, SIAM J. Comput. 1 (1972) 146-160.

[27] L.E. Trotter, Line perfect graphs, Math. Program. 12 (1977) 255-259.

[28] S. Tsukiyama, M. Idle, H. Ariyoshi, Y. Shirakawa, A new algorithm for generating all the maximal independent sets, SIAM J. Comput. 6 (1977) 505-517.

[29] W.D. Wallis, G.H. Zhang, On maximal clique irreducible graphs, J. Combin. Math. Combin. Comput. 8 (1990) 187-193.

[30] M. Yannakakis, The complexity of the partial order dimension problem, SIAM J. Algebr. Discrete Methods 3 (1982) 351-358.

[31] G. Zambelli, A polynomial recognition algorithm for balanced matrices, J. Combin. Theory Ser. B 95 (2005) 49-67. 